\title{
Polyhydroxyalkanoate Synthesis by Mixed Microbial Consortia Cultured on Fermented Dairy Manure: Effect of Aeration on Process Rates/Yields and the Associated Microbial Ecology
}

\author{
Erik R. Coats ${ }^{1}$, Benjamin S. Watson ${ }^{2}$, Cynthia K. Brinkman ${ }^{3}$
}

(1)

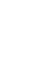

10

\footnotetext{
${ }^{1}$ Department of Civil Engineering, University of Idaho, Moscow, ID, USA 83844-1022. Correspondence concerning this paper should be addressed to him at Phone: (208) 885-7559; FAX: (208) 885-6608; email: ecoats@uidaho.edu

${ }^{2}$ Brown and Caldwell, Seattle, WA (at the time of the research, was a graduate student in the Department of Civil Engineering, University of Idaho, Moscow, ID).

${ }^{3}$ Department of Civil Engineering, University of Idaho, Moscow, ID, USA 83844-1022.
} 
Abstract.

Polyhydroxyalkanoates (PHAs) are biodegradable polymers that can substitute for petroleum-based plastics in a variety of applications. One avenue to commercial PHA production involves coupling waste-based synthesis with the use of mixed microbial consortia (MMC). In this regard, production requires maximizing the enrichment of a MMC capable of feast-famine PHA synthesis, with the metabolic response induced through imposition of aerobic-dynamic feeding (ADF) conditions. However, the concept of PHA production in complex matrices remains unrefined; process operational improvements are needed, along with an enhanced understanding of the MMC. Research presented herein investigated the effect of aeration on feast-famine PHA synthesis, with four independent aeration state systems studied; MMC were fed volatile fatty acid (VFA)-rich fermented dairy manure. Regardless of the aeration state, all

MMC exhibited a feast-famine response based on observed carbon cycling. Moreover, there was no statistical difference in PHA synthesis rates, with qPHA ranging from 0.10-0.19 CmmolPHA $\mathrm{gVSS}^{-1} \mathrm{~min}^{-1}$; VFA uptake rates exhibited similar statistical indifferences. PHA production assessments on the enriched MMC resulted in maximum intracellular concentrations ranging from $22.5-90.7 \%\left(\mathrm{mgPHA}_{\mathrm{mgSS}}{ }^{-1}\right)$; at maximum concentration, the mean hydroxyvalerate mol content was $73 \pm 0.6 \%$. While a typical feast-famine dissolved oxygen (DO) pattern was observed at maximum aeration, less resolution was observed at decreasing aeration rates, suggesting that DO may not be an optimal process monitoring parameter. At lower aeration states, nitrogen cycling patterns, supported by molecular investigations targeting AOBs and NOBs, indicate that $\mathrm{NO}_{2}$ and $\mathrm{NO}_{3}$ sustained feast-famine PHA synthesis. Next-generation sequencing analysis of the respective MMC revealed numerous and diverse genera exhibiting the potential to achieve PHA synthesis, suggesting functional redundancy embedded in the diverse MMC. Ultimately, results demonstrate that aeration can be controlled in waste-based ADF systems to sustain PHA 
42 production potential, while enriching for a diverse MMC that exhibits potential functional

43 redundancy. Reduced aeration could also enhance cost competitiveness of waste-based PHA

44 production, with potential further benefits associated with nitrogen treatment.

45

46 Key Words. Polyhydroxyalkanoates, PHA; volatile fatty acids, VFAs; aerobic dynamic feeding,

47 ADF; Next generation sequencing; oxygen mass transfer coefficient 


\section{Introduction}

Biologically-based products are of increasing interest due to their potentially lower

environmental impact relative to synthetic alternatives, as well as their foundation on renewable resources. In particular, plastics of biological origin have gathered significant attention in recent years (e.g., (Chen and Patel 2012)), with a principal focus on the production of polylactic acid (PLA), starch-based polymers, and polyhydroxyalkanoates (PHAs) (Yates and Barlow 2013). PLA is synthesized through a combination of chemical and biological processes, with corn being the principal substrate to provide the necessary sugar building blocks; while PLA is of biological origin, it exhibits generally poor biodegradability (Rudnik 2008). Thermoplastic starch (TPS) production is a chemically-based process, often involving use of a plasticizer; TPS exhibits good biodegradability (Rudnik 2008). In contrast to PLA and TPS, PHA is exclusively a biological process, and exhibits excellent biodegradability. Moreover, PHA can more universally be substituted for petro-plastics (Shen et al. 2010), with applications including films, utensils, and packaging (Madison and Huisman 1999).

Functionally, PHA is an intracellular, amorphous granule synthesized by bacteria as a carbon and energy storage reserve (Serafim et al. 2008). However, in a desiccated state PHA, is a biodegradable thermoplastic. Poly-3-hydroxybutyrate (PHB) was the first form of PHA discovered (Lemoigne 1926), with many additional structures since identified (Madison and Huisman 1999) including poly-3-hydroxyvalerate (PHV) and the copolymer PHBV. Carbon substrate dictates structure and polymeric properties (Madison and Huisman 1999). For example, PHB is synthesized with even numbered carbon substrate (e.g., acetate, glucose) and is a stiff, brittle plastic (Padermshoke et al. 2004), while PHBV is synthesized with even and odd numbered carbon substrate (e.g., acetate and propionate), exhibits reduced crystallinity and 
71 improved ductility (Luzier 1992, Padermshoke et al. 2004), and is less prone to thermal

72 degradation during processing (Luzier 1992).

Despite the intrinsic potential of this useful biopolymer, PHA production cost is

74 impeding extensive market penetration and adoption. The cost of industrially-produced PHA is

75 largely driven by the need to maintain axenic cultures and provide refined carbon substrate

76 (Fernández-Dacosta et al. 2015). Use of waste substrate can reduce the substrate cost

77 considerably (Gurieff and Lant 2007). Moreover, coupling waste-based PHA synthesis with the

78 use of mixed microbial consortia (MMC; (Dias et al. 2006, Serafim et al. 2008)) presents a

79 potentially optimal commercial model within which success can be realized, particularly

80 considering that many organic-rich waste streams are candidate substrates for the technology. In

81 this regard, the most commonly employed strategy for PHA production using MMC and waste

82 substrate is a three-stage system that comprises feedstock fermentation, enrichment of PHA-

83 producing bacteria, and PHA production (Serafim et al. 2008). The fermentation stage converts

84 organic material in waste feedstocks to volatile fatty acids (VFAs; optimal precursors for PHA

85 synthesis), while the enrichment stage sustains an MMC enriched for PHA-producing bacteria.

86 The production stage uses a fraction of the enriched culture in a fed-batch reactor to generate

87 commercial quantities of PHA. To complete the production process, the PHA-rich biomass is

88 harvested and subjected to polymer extraction and processing. Given that fermentation of most

89 waste substrates generates even and odd carbon VFAs, MMC PHA synthesis commonly yields

90 PHBV.

91 Realizing commercial PHA quantities using MMC cultured on waste substrate is

92 predicated on maximizing the enrichment of a microbial consortium capable of feast-famine

93 PHA synthesis. Feast-famine PHA synthesis is a metabolic response to aerobic dynamic feeding 
94 (ADF) conditions applied to an MMC fed an organic carbon and nutrient-rich substrate. ADF-

95 induced PHA synthesis on VFA-rich substrate is a well-established phenomenon (Dias et al. 2006, Majone et al. 1996); indeed, recent research has identified proteins associated with the

97 metabolic response (Hanson et al. 2016). For a sequencing batch reactor (SBR), ADF conditions are imposed by first providing an MMC with substrate rich in VFAs in a short duration "feast"

99 time period, thereby creating a large substrate gradient in bulk solution. Operating under aerobic conditions, the microbes rapidly convert the substrate to (predominantly) PHA. Conventionally,

101 the feast phase is defined as ending upon consumption of exogenous substrate; thereafter, 102 conditions are referred to as "famine." By combining short periods of exogenous substrate availability with long periods of exogenous substrate deficiency, PHA-producing bacteria are 104 enriched over non-PHA producing bacteria (Reis et al. 2003, van Loosdrecht et al. 1997). Additionally, the reduced anabolic capacity realized late in the famine stage impairs growth and 106 induces the storage of excess substrate as PHA in the subsequent feast phase (Dias et al. 2006, 107 Serafim et al. 2008, van Loosdrecht et al. 1997). In the Production reactor, the inoculum is exposed to a sustained feast response to maximize conversion of VFAs to PHA. The feast109 famine response is broadly recognized as the most efficient means to produce PHA using mixed microbial consortia (Serafim et al. 2008), and has been observed in multiple studies. In considering commercial ADF PHA production, a large operational expense is aeration 112 (Akiyama et al. 2003). However, little is known about aeration requirements and the associated 113 effects of reduced oxygen mass transfer (and, intrinsically, reduced residual dissolved oxygen 114 (DO)) on PHA production by MMC. A specific interest is the impact of low residual DO on 115 PHA "feast." Most ADF PHA research imposes aeration in excess such that oxygen is not a 116 limiting nutrient during the feast period. To the authors' knowledge, only one other research 
117 group has specifically assessed aeration effects on VFA (acetate) conversion to PHA (Third et al. 118 2003b, Third et al. 2004). However, while their research demonstrated that increased PHA yield 119 could be achieved through reducing the aeration rate, investigations focused on PHB synthesis 120 within the context of wastewater treatment, not commercial biopolymer production under 121 imposed ADF conditions. In a somewhat related study, Moralejo-Garate et al. (2013) examined 122 the effect of oxygen limitation on PHB production by MMC cultured on pure glycerol under 123 ADF conditions at near-mesophilic temperatures $\left(30^{\circ} \mathrm{C}\right)$; glycogen synthesis was also examined, 124 based on the potential for bacteria to store glycerol via reverse glycolysis. Results showed that 125 aeration, which was controlled based on applied oxygen mass transfer coefficients $\left(\mathrm{k}_{\mathrm{L}} \mathrm{a}\right.$; values 126 of $7.4,11.4$, and $15.6 \mathrm{hr}^{-1}$ ), adversely impacted both PHB and glycogen storage with decreasing $127 \mathrm{k}_{\mathrm{L}} \mathrm{a}$; the PHB synthesis rate and associated yield were maximized under maximal DO conditions. With an aim to contribute toward future ADF PHA commercialization, research was 129 conducted to assess and develop an enhanced understanding of the impacts of aeration on PHA 130 synthesis, yield, and production by MMC cultured on waste substrate (specifically VFA-rich 131 fermented dairy manure); as a further inquiry, the respective MMC were characterized using 132 next-generation sequencing methods. Dairy manure was selected as the target substrate based on 133 the ready availability of sufficient organic carbon for PHA synthesis and also the fact that 134 enhanced waste management practices are needed in the dairy industry to ameliorate 135 environmental challenges while concurrently enhancing industry economics (Coats et al. 2013). 136 Research objectives were to i) establish PHA Enrichment reactors at varying aeration rates 137 (established based on the oxygen mass transfer coefficient, $\mathrm{k}_{\mathrm{L}} \mathrm{a}$, with DO measured as a surrogate 138 response), ii) assess, characterize, and compare Enrichment reactor process performance relative 139 to oxygen mass transfer, iii) establish the potential effects of reduced oxygen mass transfer on 
140 ADF PHA production, and iv) evaluate and characterize the MMC ecology across imposed 141 aeration conditions.

\section{2. Materials and Methods}

2.1 PHA Enrichment Reactors. Four bench-scale PHA Enrichment reactors were

144 operated for this research (Table 1); reactor identification was based on the applied oxygen mass

145 transfer coefficient, $\mathrm{k}_{\mathrm{L}} \mathrm{a}$ (e.g., AE-4 was operated at a $\mathrm{k}_{\mathrm{L}}$ a of $4 \mathrm{hr}^{-1}$ ). All reactors were operated as

146 SBRs, were continuously stirred with no settling phase, and were operated with an SRT and

147 HRT of 4 days and cycle length of 24 hours. Aeration was accomplished with a 2.0-micron gas

148 sparger (Williams Brewing, San Leandro CA, USA); air flow rates (Table 1) were controlled

149 using Aera PI-98 mass flow controllers (MFC's) rated to 0-1000 \pm 1 standard cubic cm per min

150 (sccm) (Hitachi Metals America, San Jose, California, USA). Mixing was achieved with Thermo

151 Scientific Cimarec magnetic stir plates (Thermo Fisher Scientific Inc., Waltham, MA, USA) and

$1523.8 \mathrm{~cm}^{\text {Teflon }}{ }^{\mathrm{TM}}$ coated magnetic stir bars. The reactors were scrubbed daily; the aeration stones

153 were soaked in $1 \mathrm{~N} \mathrm{HCl}$ for a minimum of 5 min each day, rinsed with deionized water, cleaned

154 with Kimwipe $\odot$, and then re-submerged in the reactor. After reactor cleaning, $300 \mathrm{~mL}$ of mixed

155 liquor was removed and either disposed of or used in a PHA Production reactor. Each reactor

156 was then re-filled with $300 \mathrm{~mL}$ of substrate comprised of $40 \%$ dairy manure fermenter liquor and

$15760 \%$ tap water by volume; the resultant organic loading rate was $0.44 \pm 0.11 \mathrm{gCOD}_{\mathrm{VFA}} \mathrm{L}^{-1} \mathrm{~d}^{-1}$

158 (12.5 $\left.\pm 3.2 \mathrm{Cmmol} \mathrm{L}^{-1} \mathrm{~d}^{-1} ; \mathrm{n}=19\right)$. The applied OLR was approximately an order of magnitude

159 lower than Dionisi et al. (2006) but comparable to that of Dionisi et al. (2001, 2004). For this

160 study, batches of substrate were mixed every three days and stored at $4^{0} \mathrm{C}$ until use. Tap water

161 was added prior to feeding and cleaning to compensate for evaporation. Enrichment reactors

162 were operated at ambient room temperature $\left(22-25^{\circ} \mathrm{C}\right)$. 
commonly reported as volume of air provided per reactor volume or as a surrogate based on DO concentration, while some just report the equipment used. Such approaches are acceptable when

166 PHA reactors are excessively aerated to ensure oxygen is not limiting the targeted biochemical

167 reaction; this condition is typical for most PHA investigations. However, properly quantifying

168 aeration characteristics is important if the research is to be ultimately transferred to full-scale, 169 particularly considering that aeration costs must be minimized.

Aeration in this study was controlled based on the universal oxygen mass transfer 171 coefficient, $\mathrm{k}_{\mathrm{L}} \mathrm{a}$. Oxygen uptake rate (OUR) and $\mathrm{k}_{\mathrm{L}} \mathrm{a}$ were determined in accordance with the 172 dynamic degassing method (Garcia-Ochoa and Gomez 2009). The $\mathrm{k}_{\mathrm{L}} \mathrm{a}$ was measured during the 173 famine phase of the Enrichment reactor cycle to prevent VFA catabolism from influencing the 174 OUR. As part of the $\mathrm{k}_{\mathrm{L}}$ a quantification, air flow to the reactor was first stopped and the DO was 175 allowed to decrease to approximately $2 \mathrm{mg} \mathrm{L}^{-1}$. Once the DO reached $2 \mathrm{mg} \mathrm{L}^{-1}$, air flow was 176 returned to the reactor and the DO concentrations recorded. The $\mathrm{k}_{\mathrm{L}}$ a coefficient with the best fit

177 was quantified using the residual squared sum of errors (with an $\mathrm{R}^{2}$ greater than 0.999 ). $\mathrm{k}_{\mathrm{L}}$ a was 178 corrected for temperature based on the van't Hoff-Arrhenius relationship using $Q=1.024$

179 (Tchobanoglous et al. 2014). Operational $\mathrm{k}_{\mathrm{L}}$ a values for all Enrichment reactors were confirmed 180 every two weeks, at a minimum; in addition, prior to process assessment, $\mathrm{k}_{\mathrm{L}}$ a values were 181 confirmed both seven and one day prior to testing.

2.3 Effects of Wastewater Characteristics on Aeration. The relative oxygen 183 transfer efficiency of each reactor was quantified based on the parameter $\alpha$, normalizing the $\mathrm{k}_{\mathrm{L}} \mathrm{a}$ 184 for wastewater to the $\mathrm{k}_{\mathrm{L}}$ a for tap water (Tchobanoglous et al. 2014). To quantify $\alpha$, the 185 wastewater $\mathrm{k}_{\mathrm{L}}$ a was first corrected to $20^{\circ} \mathrm{C}$. The tap water $\mathrm{k}_{\mathrm{L}}$ a was measured in the same reactor 
and with the same aeration apparatus; sodium sulfite and cobalt chloride were used to remove oxygen from solution. $\alpha$ values were 1.1 (AE-4), 0.88 (AE-8), 1.01 (AE-12), and 0.98 (AE-20), respectively.

\subsection{PHA Production Reactors. All PHA Production reactors had an initial} operational volume of $300 \mathrm{~mL}$ (i.e., the daily waste from the respective Enrichment reactors), and investigations were conducted using undiluted dairy manure fermenter liquor. The same aeration stones and mixing plates were used as in the Enrichment reactors. During operation of the PHA Production reactors, DO was monitored continuously. The aeration rate was not controlled; instead, air flow rate was set so that DO concentration exceeded $1.0 \mathrm{mg} \mathrm{L}^{-1}$, such that oxygen was not a limiting nutrient in the biochemical reactions.

The PHA production potential of inocula obtained from the Enrichment reactors was evaluated in four discrete stages (identified as 1-A, -B, followed by 2-A, -B). For stage 1-A and 1-B operations, the PHA Production reactors were pulsed with substrate following a spike in residual DO or at a set time interval (45 min for 1-A, 30 min for 1-B) if DO did not otherwise indicate a need for more substrate. The time interval selection was based on the previous day Enrichment reactor VFA uptake rate and VFA concentration of the Production reactor substrate. Each pulse volume was held volumetrically constant at $10 \%$ of reactor volume at time of pulse. Operational stages 2-A and 2-B employed a 30 min feeding interval, with $30 \mathrm{~mL}$ of substrate supplied per pulse. The mass of VFAs added for each interval in stages 2-A and -B was designed to ensure that the empirical maximum bulk solution VFA concentration of $60 \mathrm{Cmmol} \mathrm{L}^{-1}$ (Serafim et al. 2004) was not exceeded.

2.5 Source of Manure; Dairy Manure Fermenter Operations. Raw dairy manure was collected from the University of Idaho dairy every 7 to 14 days, sampled for total solids (TS) 
and volatile solids (VS) content at the time of collection, and stored at $4^{\circ} \mathrm{C}$ until use. Manure was collected to minimize contamination with refractory lignocellulosic bedding material.

The fermented dairy manure liquor for the PHA investigations was obtained from a bench-scale dairy manure fermenter; manure characteristics and fermenter operational conditions are detailed in Stowe et al. (2015). Briefly, a 22.7 L fermenter was operated at a volume of $20 \mathrm{~L}$, a $4 \mathrm{~d}$ SRT and HRT, and a target OLR of $8.75 \mathrm{gVS} \mathrm{L}^{-1} \mathrm{~d}^{-1}$, with feeding and wasting conducted once per day. The target OLR was maintained based on the VS samples collected from the raw manure. Fermentation took place at room temperature $\left(22-25^{\circ} \mathrm{C}\right)$. Fermenter mixing was accomplished using a $9.5 \mathrm{~cm}$ diameter helical impeller driven by an Oriental Motor (USM315401W 15 W AC; San Jose, CA, USA) connected to a 3GN35SA reduction gearbox. Fermenter liquor was obtained via centrifugation of the fermenter effluent at $8000 \mathrm{rpm}$ for $5 \mathrm{~min}$ at room temperature to separate liquid and solid fractions. Fermenter liquor characteristics for the period of operations associated with this study were as follows ( $\mathrm{n}=26)$ : $2410 \pm 470 \mathrm{mgCOD}_{\mathrm{HAc}} \mathrm{L}^{-1}$, $1230 \pm 1720 \mathrm{mgCOD}_{\mathrm{HPr}} \mathrm{L}^{-1}, 982 \pm 238 \mathrm{mgCOD}_{\mathrm{HBu}} \mathrm{L}^{-1}, 456 \pm 53 \mathrm{mgCOD}_{\mathrm{HVa}} \mathrm{L}^{-1}$, and $41 \pm 27$ $\mathrm{mgCOD}_{\mathrm{HCa}} \mathrm{L}^{-1}$. In a previous study (Hanson et al. 2016) the VFA fraction (COD basis) of dairy manure fermenter liquor was estimated at 70\%; based on Enrichment reactor effluent data, the non-biodegradable fraction was approximately 10\% (Passero et al. 2014). Limited analysis on nutrients showed ammonia-N ranging from $448-1143 \mathrm{mgN} \mathrm{L}^{-1}$ and orthophosphate ranging from $25-131 \mathrm{mgP} \mathrm{L}^{-1}$; thus, the fermenter liquor substrate was not macronutrient deficient.

2.6 Analytical Techniques. PHA content was analyzed as methyl ester derivative through GC/MS, applying a modification of the method originally proposed (Braunegg et al. 1978). Samples were treated with $1 \mathrm{~mL}$ of commercial sodium hypochlorite to lyse the cells, then centrifuged at 10,000 rpm for $4 \mathrm{~min}$. Supernatant was removed and the remaining solids 
232 rinsed with deionized water twice. Biomass was dried at $100-105^{\circ} \mathrm{C}$ for a minimum of $24 \mathrm{hr} .10$ -

$23320 \mathrm{mg}$ of dried biomass was weighed into a test tube for digestion along with $2 \mathrm{~mL}$ of methanol

234 acidified with $\mathrm{H}_{2} \mathrm{SO}_{4}$ (3\% vol basis) and $1 \mathrm{~mL}$ of chloroform with $0.5 \mathrm{mg} \mathrm{L}^{-1}$ benzoic acid

235 (internal standard). The sample was digested for $4 \mathrm{hr}$ at $100^{\circ} \mathrm{C}$ in a Hach DRB 200 digestion

236 block (Hach Company, Loveland CO, USA). Samples were allowed to cool before adding $2 \mathrm{~mL}$

237 of deionized water, then vortexed for $30 \mathrm{~s}$. The samples stabilized for a minimum of 5 min to

238 allow the organic and aqueous phases to separate. The organic phase was pipetted and filtered

239 through a Pasteur pipette column of anhydrous sodium sulfate into a $2 \mathrm{~mL}$ screw top glass vial

240 with Teflon®-sealed cap (Thermo Fisher Scientific Inc., Waltham, MA, USA). Samples were

241 injected into a ThermoQuest Trace ${ }^{\mathrm{TM}} \mathrm{GC}$ with a Finnigan PolarisQ iontrap using a ThermoQuest

242 AS2000 autosampler (Thermo Fisher Scientific Inc., Waltham, MA, USA). The inlet was

243 operated in split mode, with a temperature of $210^{\circ} \mathrm{C}$. Separation was achieved using a ZB1 (30 m

$244 \times 0.25 \mathrm{~mm}$ ID) capillary column (Phenomenex, Torrance, CA, USA) which was ramped from an

245 initial $40{ }^{\circ} \mathrm{C}$ to $200{ }^{\circ} \mathrm{C}$ at $5^{\circ} \mathrm{C} \mathrm{min}^{-1}$. 3-hydroxybutyrate and 3-hydroxyvalerate monomers were

246 verified by mass spectra at $103 \mathrm{~m} / \mathrm{z}$ and retention time matching based on commercial standards

247 (Sigma-Aldrich Co., St. Louis, MO, USA). The standard curves for quantification had

248 correlation coefficients of $\mathrm{R}^{2}>0.95$ (3-Hydroxyvalerate) and $\mathrm{R}^{2}>0.98$ (3-Hydroxybutyrate).

249 VFAs (acetic, propionic, butyric, isobutyric, valeric, isovaleric, and caproic acids) and

250 methanol were quantified using a Hewlett-Packard 6890 series gas chromatograph (GC) (Agilent

251 Technologies, Inc., Santa Clara, CA, USA) equipped with a flame-ionization detector (FID) and

252 a Hewlett-Packard 7679 series injector. The system was interfaced with the Hewlett-Packard GC

253 ChemStation software version A.06.01. VFA separation was achieved using a capillary column

254 (Heliflex ${ }^{\circledR} \mathrm{AT}^{\mathrm{TM}}$-AquaWax-DA, $30 \mathrm{~m}$ x $0.25 \mathrm{~mm}$ ID, W. R. Grace \& Co., Deerfield, IL, USA) 
255 which was ramped from an initial $50^{\circ} \mathrm{C}$ to $200^{\circ} \mathrm{C}$ in three steps $\left(2 \mathrm{~min}\right.$ at $50^{\circ} \mathrm{C}$, ramp to $95^{\circ} \mathrm{C}$ at

$25630^{\circ} \mathrm{C} \mathrm{min}^{-1}$ then to $150^{\circ} \mathrm{C}$ at $10^{\circ} \mathrm{C} \mathrm{min}^{-1}$ and hold for $3 \mathrm{~min}$; finally, ramp to $200^{\circ} \mathrm{C}$ at $25^{\circ} \mathrm{C} \mathrm{min}^{-1}$

257 and hold for $12 \mathrm{~min})$ with helium as the carrier gas $\left(1.2 \mathrm{~mL} \mathrm{~min}^{-1}\right)$. The split/splitless injector

258 and detector were operated isothermally at 210 and $300^{\circ} \mathrm{C}$, respectively. Prior to analysis,

259 samples were acidified to a $\mathrm{pH}$ of 2 using nitric acid. $0.5 \mu \mathrm{L}$ of each sample was injected in 20:1

260 split mode. VFA concentrations were determined through retention time matching with known

261 standards (Sigma-Aldrich Co., St. Louis, MO, USA; Thermo Fisher Scientific Inc., Waltham,

262 MA, USA) and linear standard curves $\left(\mathrm{R}^{2}>0.99\right)$.

263 For soluble constituents, samples were first centrifuged to remove solids/biomass and 264 then filtered through a $0.22 \mu \mathrm{m}$ syringe filter (Millipore Corp., Billerica, MA, USA). Soluble

$265 \mathrm{NO}_{3}-\mathrm{N}$ was determined in accordance with Hach method 10020 . Soluble $\mathrm{NH}_{4}-\mathrm{N}$ testing followed

266 Hach method 10031. Soluble $\mathrm{NO}_{2}-\mathrm{N}$ was determined using Hach method 8153 and method

267 8507. A Spectronic ${ }^{\circledR} 20$ Genesys $^{\mathrm{TM}}$ spectrophotometer (Thermo-Fisher Scientific Corp,

268 Waltham, MA, USA) was utilized to measure the absorbance of the reacted sample. Soluble

269 reactive phosphorus was determined in accordance with Hach (Loveland, CO, USA) method

2708048 (method equivalent to Standard Methods 4500-PE (Clesceri LS 1998)). Nutrient

271 concentrations were determined utilizing appropriate standard curves $\left(\mathrm{R}^{2}>0.99\right)$.

272 Dissolved oxygen (DO) was measured using a Hach IntelliCAL ${ }^{\text {TM }}$ LDO101 probe

273 connected to a Hach HQ40d Multi-Parameter Meter. Data was logged using the USB and AC

274 power adapter. TS and VS were measured following Standard Methods 2540D and 2540E

275 respectively (APHA et al. 2012).

276

2.7 Quantitative Polymerase Chain Reaction (qPCR). qPCR was applied to

277 estimate the relative abundance of ammonia oxidizing bacteria (AOB) and nitrite oxidizing 
278 bacteria (NOB) in the Enrichment reactors. Bulk genomic DNA from each reactor was extracted 279 using a PowerSoil@ DNA Extraction Kit (MO BIO Laboratories Inc., Carlsbad, CA USA).

280 Genomic DNA yield and purity was quantified using a Synergy H1 Multi-Mode Reader (BioTek, 281 Winooski, VT). qPCR was conducted on a StepOne Plus ${ }^{\mathrm{TM}}$ Real-Time PCR system (Applied 282 Biosystems, Foster City, CA) using iTaq ${ }^{\mathrm{TM}} \mathrm{SYBR}^{\circledR}$ Green Supermix w/ROX (Bio-Rad 283 Laboratories, Inc., Hercules, CA, USA) and a total reaction volume of $25 \mu 1$. Eubacteria were 284 amplified using primer sets developed by Muyzer et al. (1993). Amplification of AOBs was 285 based on a primer set for the gene ammonia monooxygenase (amoA) (Rotthauwe et al. 1997). 286 For NOBs, Nitrobacter spp. and Nitrospira were amplified using 16S rDNA sequences. qPCR 287 settings were in accordance with Winkler et al. (2011). AOB, Nitrobacter, and Nitrospira 288 abundance relative to eubacteria was estimated using the mean efficiencies for each primer set 289 and the Cq values for the individual samples, assuming average $16 \mathrm{~S}$ rDNA gene copy numbers 290 of 4.1 for eubacteria, 2.5 for AOB (Leininger et al. 2006), and 1.0 for both Nitrobacter and 291 Nitrospira (McIlroy et al. 2015). All samples were assessed in triplicate with $5 \mathrm{ng}$ of total 292 genomic DNA per reaction. qPCR melting curves were evaluated to confirm a single melting 293 peak, and agarose gel analysis confirmed a single band for each primer set. Amplification 294 efficiencies were calculated for each primer set using baseline-corrected fluorescence data 295 (StepOne software v2.0), and the LinRegPCR program (Ramakers et al. 2003). The cycle 296 threshold was set at a constant value across all samples based on location within the log-linear 297 region for determination of $\mathrm{Cq}$ values (cycle number at which the measured fluorescence 298 exceeds the cycle threshold). was performed on genomic DNA recovered from the respective PHA Enrichment reactors on 
301 three operational days. Bacterial 16S rRNA gene fragments were amplified and sequenced in 302 accordance with Hanson et al. (2016). DNA amplicons were generated using two PCR rounds 303 (round one amplified the targeted region of the 16S rRNA gene and round two attached 304 sequencing adapters and sample barcodes) for eubacteria (Shen et al. 2016); the primers are 305 described in Hanson et al. (2016). The barcoded amplicons were sequenced using an Illumina 306 MiSeq instrument creating paired end 2x300 bp libraries (Illumina, Inc., San Diego, CA). Sequence analysis and taxonomic classification were performed following Hanson et al. 308 (2016). Briefly, the Illumina MiSeq reads were demultiplexed and assigned to expected barcode 309 and primer sequences using the Python script dbcAmplicons

310 (https://github.com/msettles/dbcAmplicons). After the primer sequences were trimmed, the reads 311 were joined into a single amplicon sequence using the application FLASH (Magoč and Salzberg 312 2011). The Ribosomal Database Project (RDP) Naïve Bayesian classifier was then used to assign 313 the joined sequences to phylotypes (Wang et al. 2007); assignment was made to the lowest 314 taxonomic rank with a bootstrap score $\geq 50 \%$. The relative abundance of individual phylotypes 315 in each sample was determined as the percentage of the corresponding sequence reads among the 316 total sequence reads in the sample.

$317 \quad 2.9 \quad$ Data Analysis. Custom software (altvisngs; available at

318 https://github.com/nguho/altvisngs) was used to quantify the sample diversity and evenness

319 indices, complete the rarefaction and hierarchical cluster analysis, and generate the taxon 320 hierarchy with relative abundance, heatmap, and summary bar plot images (see Supplementary 321 Data). Single factor ANOVA was used to establish differences in means using Microsoft Excel, 322 with significance declared at $p<0.05$.

\section{$323 \quad 3 . \quad$ Results and Discussion}


The performance of mixed microbial consortia (MMC) in each unique aeration-state

325 PHA Enrichment reactor was evaluated for both PHA synthesis and production potential. The

326 PHA Enrichment reactors were operated for a period of 271 days, with detailed performance

327 interrogations conducted on operational days 19, 52, 144, 190, and 271. In addition, PHA

328 production evaluations were conducted on operational days 173, 238, 263, and 271. Results from

329 the Enrichment reactor investigations are presented and discussed below first, with Production

330 evaluations presented thereafter.

3.1 Aeration Effects on PHA Synthesis. As described, the PHA Enrichment reactors were operated identically with the exception of the experimental variable: the aeration rate, which was established based on the applied oxygen mass transfer coefficient, $\mathrm{k}_{\mathrm{L}} \mathrm{a}$, and measured based on residual DO. Four aeration rates were examined: $\mathrm{k}_{\mathrm{L}} \mathrm{a}=4,8,12$, and $20 \mathrm{hr}^{-1}$. While these applied aeration rates aligned with a comparative study of Third et al. ((2003b); $\left.\mathrm{k}_{\mathrm{L}} \mathrm{a}=6-51 \mathrm{hr}^{-1}\right)$

336 and were similar to a PHB-focused study that used a differing substrate (glycerol) (Moralejo-

337 Gárate et al. 2013), applied values were less than the minimum $\mathrm{k}_{\mathrm{L}} \mathrm{a}$ of $120 \mathrm{hr}^{-1}$ recommended by

Dias et al. (2006) for MMCs producing PHA on waste substrate.

First considering VFA utilization patterns - which, coupled with PHA synthesis patterns,

340 indicate the presence or absence of an induced feast-famine response - results confirm the 341 targeted response was in fact induced regardless of the applied aeration state (Fig. 1). In all 342 Enrichment reactors the time required for complete VFA utilization ranged from approximately 34350 min to approximately $120 \mathrm{~min}$, which is consistent with similar ADF PHA studies (Beun et al. 344 2002, Serafim et al. 2004). More importantly, PHA synthesis patterns were comparable across 345 the four enrichment reactors (Figs. 2a-2d). Ultimately, the observed PHA synthesis on VFAs 346 under the applied ADF conditions and across the four aeration states was consistent with feast- 
347 famine theory (Dias et al. 2006). All consortia exhibited a maximum PHA concentration

348 approaching 10-15 $\mathrm{Cmmol} \mathrm{L}^{-1}$ and comparable lowest peak concentrations of approximately 3-5

$349 \mathrm{Cmmol} \mathrm{L}^{-1}$. Average intracellular concentrations $\left(\mathrm{gPHA}_{\mathrm{gTSS}}{ }^{-1}\right)$ were similar across aeration

350 states, as was the variability over the tested operational days. Comparing results, there was no

351 statistical difference in average PHA yield, although it does appear that VFA conversion to PHA

352 was more efficient under higher aeration states (Table 2).

DO has been suggested as a surrogate parameter that could be monitored in real-time to

354 establish the end of the PHA feast period (Beun et al. 2002, Dias et al. 2005, Serafim et al.

355 2004); the importance of this real-time feedback relates to downstream PHA production, in that

356 DO could be used as an indicator that famine conditions are impending and thus more VFAs are

357 needed to sustain the feast and maximize PHA synthesis. Examining DO for all Enrichment

358 reactors revealed important results (Fig. 2). A typical ADF response would exhibit a DO "valley"

359 during the feast phase and immediately after receiving new substrate, followed by a rapid

360 increase in residual DO as the MMC enters a famine period (and the microorganisms can more

361 readily manage available electrons for growth). Indeed, the biomass realizing the highest aeration

362 rate (AE-20) exhibited a typical feast-famine DO response (Fig. 2a), with both initial ( $\mathrm{t}=0$ ) and

363 famine residual DO concentrations approaching saturation, while the DO concentration during

364 the feast phase averaged $1.25 \pm 0.99 \mathrm{mg} \mathrm{L}^{-1}$. At the other aeration extreme (reactor AE-4; Fig. 2d),

365 atypical DO conditions were observed. Specifically, while a DO "valley" was observed, the

366 residual DO remained generally less than $2 \mathrm{mg} \mathrm{L}^{-1}$ well beyond the end of the feast period

367 (which occurred consistently less than 150 min into the operational cycle; Fig. 1d) and thus did

368 not strongly signal onset of famine conditions. While the DO ultimately increased to

369 approximately $6.5 \mathrm{mg} \mathrm{L}^{-1}$ at the end of the SBR cycle (data not shown), the typical feast-famine 
DO pattern was not observed. Similarly, the middle two aeration rates exhibited atypical feast-

371 famine DO profiles, in that the DO more slowly increased during the famine period (but also

372 ultimately realized an end of cycle DO ranging from 5.7-7 $\mathrm{mg} \mathrm{L}^{-1}$ ). Results thus suggest that,

373 depending on the imposed aeration state, DO may not be an ideal candidate for real-time process

374 monitoring of the targeted feast-famine response.

375 Process kinetics (VFA consumption and PHA synthesis rates (Table 3)) were interrogated

376 to illuminate potential impacts of aeration rate on the PHA Enrichment reactor biomass. First

377 considering VFA consumption rates, the average rate of VFA utilization $\left(\mathrm{r}_{\mathrm{VFA}}\right)$ ranged from 0.20

378 (AE-12) to 0.31 (AE-20) CmmolVFA L ${ }^{-1} \mathrm{~min}^{-1}$, while the specific rate of VFA utilization (qvFA)

379 ranged from 0.14 (AE-12) to 0.26 (AE-20) $\mathrm{CmmolVFA} \mathrm{gVSS}^{-1} \mathrm{~min}^{-1}$. Regarding PHA synthesis,

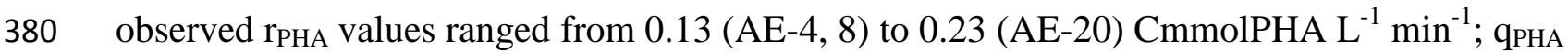

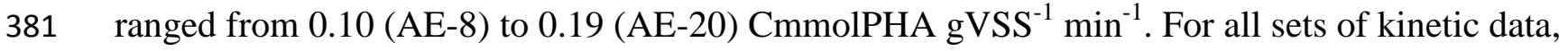

382 there was no statistical difference between the respective averages. Moreover, specific rates were

383 higher than observed by Third et al. (2003b) for comparable aeration rates. Third et al. (2003b)

384 observed a residual DO during VFA feast of $0 \mathrm{mg} \mathrm{L}^{-1}$; conversely, microaerophilic conditions

385 were generally maintained in this study (Fig. 2). Certainly the lack of DO during "feast" in the

386 study by Third et al. (2003b) could have adversely impacted the rates in question. Comparing

387 results with studies wherein aeration induced near-saturation conditions (as summarized in Beun

388 et al. (2002) and observed by Serafim et al. (2004)), this study's qVFA and qPHA values were

389 within the lower observed range (although these other referenced studies used pure acetate as

390 substrate, not a complex waste substrate), while yield values were comparable. While $\mathrm{k}_{\mathrm{L}} \mathrm{a}$ was

391 varied across the Enrichment reactors, more importantly during the carbon flux feast period the

392 residual DO across the four Enrichment reactors did not vary substantially (Fig. 2), which likely 
explains why the respective rates exhibited no statistical differences. Although Third et al. (2003b) observed that VFA utilization rates and PHA synthesis rates decrease with reduced $\mathrm{k}_{\mathrm{L}} \mathrm{a}$, in comparison with this study, fully anaerobic conditions were realized at the lowest $\mathrm{k}_{\mathrm{L}} \mathrm{a}$ 's.

Although there was no statistically significant difference between observed carbon flux and utilization rates across the imposed aeration states, the average highest rates (for both VFA uptake and PHA synthesis) occurred in the reactor with the greatest aeration rate (reactor AE20). Conversely, the MMC in reactor AE-4 (lowest $\mathrm{k}_{\mathrm{L}} \mathrm{a}$ ) exhibited the lowest uptake rates.

Overall, the VFA and PHA specific rates were variable over time, potentially due to changes in bacterial populations (see also Section 3.5). However, specific PHA synthesis rates by MMCs have been commonly observed to be quite variable, and rates reported herein are well within observed ranges (Beun et al. 2002, Reis et al. 2003).

One final metric to assess the relative magnitude (and to a certain degree efficiency) of a feast-famine response, as well as the successful enrichment of PHA-producing bacteria, is the feast-to-famine (F:F) ratio (van Loosdrecht et al. 1997). The F:F ratio is the total length of the feast phase compared to the total length of the operational cycle; a F:F value of 0.2 has been suggested as a potential maximum to sustain a microbial consortium that will accomplish excess PHA storage over growth (Dionisi et al. 2007). All Enrichment reactors realized a F:F ratio less than 0.2 , with AE-4, $-8,-12$, and -20 operating at average $F: F$ ratios of $0.071,0.068,0.051$, and 0.037, respectively. The highest aeration rate Enrichment reactor realized the lowest $\mathrm{F}: \mathrm{F}$ ratio, which aligned with the highest observed average VFA utilization rate; considering that AE-20 was operated under the more optimal (relative) redox state, such results would be expected. In summary, reduced aeration rate exhibited limited impact on VFA utilization or PHA synthesis (kinetically or stoichiometrically) by MMC on the dairy manure-derived VFA-rich 
416 substrate in the Enrichment reactors. These findings suggest the opportunity to realize cost

417 savings (and environmental benefit) from reduced aeration for process scale-up. However, before

418 such conclusions can be firmly established, PHA production potential using inocula from the

419 Enrichment reactors must be considered.

\subsection{Impact of Enrichment Reactor Aeration Rate on PHA Production. In}

421 considering commercial PHA production, one of the more important process outcomes is peak

422 intracellular PHA content, measured on a dry weight basis and typically expressed as a

423 percentage. PHA purification requires a variety of solvents, depending on the process selected;

424 thus, higher intracellular PHA concentrations will reduce the cost of the PHA per kg produced

425 through reduced chemical usage (Dias et al. 2006, Van Wegen et al. 1998). Examining

426 intracellular PHA concentrations (Table 4), observed values (mgPHA mgTSS${ }^{-1}$, \% basis) for AE-

$4274,-8,-12$, and -20 ranged from 27.8-40.5, 18.3-54.7, 23.3-70.9, 28.3-62.6 (averages of 35.5,

$42837.4,45.9$, and 41.7, respectively); results are also shown on a VSS basis. While intracellular

429 concentrations were variable within and across the PHA Production reactors, statistically

430 (ANOVA) the Enrichment reactor aeration rate exhibited no impact $(\mathrm{p}=0.77)$. Of note, the

431 highest obtained value of $90.7 \%$ (VSS basis) exceeds reported values of $75 \%$ (Albuquerque et al.

432 2010), 77\% (Jiang et al. 2012), and 78.5\% (Serafim et al. 2004). These other cited experiments

433 were conducted under growth limitation, not in the presence of excess nutrients; moreover, the

434 latter was performed with synthetic feed. Conversely, the research presented herein was

435 conducted on real waste and without any growth limitation (ammonia, phosphorus, oxygen, and

436 substrate all in excess). Valentino et al. (2015) is the closest comparison to this work; they

437 reported VSS weight percent ranging from 50-70\%. Results from this research are comparable,

438 even outperforming, although with a larger range of realized \%PHA content. 
In addition to intracellular PHA concentration, the fractional HV content in the polymer

440 is an important metric; increased HV content reduces polymer crystallinity and improves

441 ductility (Luzier 1992, Padermshoke et al. 2004) while also rendering the PHA less prone to

442 thermal degradation during processing (Luzier 1992) and expanding commercial applications

443 (Dias et al. 2006). Mean HV content (mol basis) over the duration of all the production

444 evaluations was $0.46 \pm 0.16(n=46), 0.46 \pm 0.18(n=44), 0.49 \pm 0.18(n=45)$, and $0.49 \pm 0.19(n=46)$

445 for Production reactors AE-4, -8, -12, and -20, respectively; relative HV content within each

446 production assessment is summarized in Table 4. Interestingly, the average HV content was

447 markedly higher in the Production reactor biomass (Table 4) as compared with the Enrichment

448 reactor biomass (Table 2). A closer review of the data indicates that the HV content steadily

449 increased over the duration of a production assessment, and in many cases the HV content during

450 the initial stages of the production campaign were more consistent with that of the associated

451 Enrichment reactor. While a clear explanation on the phenomenon of increasing $\mathrm{HV}$ content is

452 not known, considering the complex VFA matrix and the associated metabolisms for conversion

453 of VFAs to PHA (Braunegg et al. 1998), it is plausible that over the production period the MMC

454 more readily oxidized acetate (via acetyl CoA and the TCA cycle), leaving the less readily

455 metabolized VFAs (C3-C5) utilized for PHV synthesis.

456 As a final analysis regarding the potential impact of Enrichment reactor aeration on PHA

457 production, the mass of carbon required to achieve maximum intracellular PHA concentration

458 was evaluated. This metric was determined at the time point when maximum intracellular PHA

459 concentration was observed, and considers the total VFAs consumed to achieve the peak PHA

460 value. The premise of this interrogation was that the lower aeration state in the Enrichment

461 reactor could render the inocula less metabolically conditioned for hyper-PHA synthesis, and 
462 thus potentially more VFA-carbon would be consumed to achieve maximal intracellular PHA

463 concentration. Indeed, a review of the data (Table 4) suggests that PHA production using inocula

464 from AE-4 (lowest aeration state) did in fact require more carbon than the higher aeration states

465 (0.46-0.78 CmmolPHA CmmolVFA ${ }^{-1}$ for AE-4 vs. 0.76-0.97 $\mathrm{CmmolPHA} \mathrm{CmmolVFA}^{-1}$ for AE-

466 20). Moreover, statistically (ANOVA) the four discrete Production reactor average yields were

467 not equal ( $\mathrm{p}=0.048$; although the averages for $\mathrm{AE}-8,-12$, and -20 were statistically equal

$468(\mathrm{p}=0.24))$. While yield values approaching or exceeding one may seem implausible, such results

469 are not uncommon when using complex wastewater substrates, as summarized by Reis et al.

470 (2003). Moreover, given the complexity of manure (Stowe et al. 2015) and associated upstream

471 process (fermentation), other forms of soluble carbon (e.g., carbohydrates; long chain fatty acids)

472 were likely present in the fermenter liquor (but not measured) which could have been converted 473 to PHA.

$474 \quad 3.3 \quad$ Assessing PHA Production Operational Strategy. Two different operational

475 methods were evaluated to maximize PHA production using MMC inocula from the Enrichment

476 reactors. The approach to PHA production is to supply excess carbon substrate to a MMC

477 capable of feast-famine PHA synthesis such that a "feast" response is sustained, thereby

478 achieving maximal intracellular PHA accumulation and associated biomass PHA production.

479 Sustaining a "feast" response requires avoiding both substrate inhibition and limitation.

481 4) the method wherein VFAs were pulsed to the biomass every 30 minutes (Fig. 4) resulted in

482 sustained VFA availability to the MMC and ultimately the highest intracellular PHA

483 concentrations (Table 4; Stages 1 vs. Stages 2 results). The relative HV content was not affected

484 by operational strategy or inocula source, as HV content was similar across all reactors for each 
485

486

487

488

489

490

491

492

493

494

495

496

497

498

499

500

501

502

503

504

505

506

507

production assessment (Table 4). The primary difference between the two operational strategies can be considered as being proactive vs. reactive, related to avoiding a substrate limitation. Applying a DO-based strategy (pulse VFAs when the residual DO increases rapidly) can be considered a reactive strategy, in that substrate is added when the bulk solution concentration is effectively exhausted. As such, depending on the process monitoring and control rigor, for a short duration the microbes can shift reliance to PHA for growth and maintenance. Indeed, such a response can be observed in the data presented in Fig. 3. To avoid PHA consumption while minimizing process control requirements, new substrate can simply be provided to the biomass on a more regular interval in order to sustain a feast response. For this study, 30 min feed intervals facilitated an environment wherein no substrate limitation was realized (Fig . 4). Moreover, the applied operational strategy (feed interval coupled with mass of VFAs added) sustained bulk solution VFA concentrations within the empirical limit to avoid PHA synthesis inhibition (maximum of $60 \mathrm{Cmmol} \mathrm{L}^{-1}$ according to Serafim et al. (2004)). Consequently, improved PHA accumulation was sustained.

3.4 Aeration Rate, Feast-Famine PHA Synthesis, and Nitrogen Cycling. To better understand and potentially explain why reduced aeration rate did not impact PHA synthesis and production, nitrogen cycling patterns in the Enrichment reactors were examined. Theoretically, $\mathrm{NO}_{2}$ and $\mathrm{NO}_{3}$ (produced through nitrification) could sustain the catabolic and anabolic metabolisms necessary for VFA feast and PHA synthesis; use of $\mathrm{NO}_{2} / \mathrm{NO}_{3}$ in the presence of oxygen has been referred to as simultaneous nitrification-denitrification (SND). Note that Enrichment reactor operations did not attempt to actively control or inhibit nitrification, and the fermented dairy manure liquor contained ammonia. Third et al. (2003a) observed SND within a feast-famine response, but operational conditions were such that $\mathrm{NO}_{3}$ was not available until the 
508 famine phase and thus PHB served as the carbon and electron source; thus, Third et al. (2003a)

509 did not observe SND during the feast phase. However, their results suggested that SND could be

510 realized by a PHA producing MMC at DO concentrations less than $1.0 \mathrm{mg} \mathrm{L}^{-1}$; research

511 presented herein met this DO limit (Fig. 2).

512

First considering Enrichment reactor AE-20 (highest aeration rate), indeed a significant

513 amount of ammonia oxidation occurred; representative results are presented in Fig. 5a, showing

514 that effluent ammonia (previous cycle) was less than $3 \mathrm{mgN} \mathrm{L}^{-1}$ and that nitrification occurred

515 throughout the operational cycle. Regarding $\mathrm{NO}_{2}$ and $\mathrm{NO}_{3}$, results (Fig. 5a) suggest that SND

516 during the feast period was not realized at the imposed residual DO concentrations. However,

517 nitrification was partially inhibited (i.e., partial nitritation $\left(\mathrm{NH}_{4} \rightarrow \mathrm{NO}_{2}\right)$; Fig. 5a), in that $\mathrm{NO}_{2}$

518 concentrations increased more significantly than $\mathrm{NO}_{3}$ for the first 6-7 hours of the operational

519 cycle. Nevertheless, late-cycle nitrification occurred in AE-20, as illustrated by the near-zero

520 preceding-cycle $\mathrm{NO}_{2}$ concentrations and correspondingly high $\mathrm{NO}_{3}$ concentrations (Fig. 5a).

521 Examining the other aeration extreme (AE-4; Fig. 5b), while again significant ammonia

522 oxidation occurred over the operational cycle, in contrast to AE-20, some $\mathrm{NO}_{2}$ and $\mathrm{NO}_{3}$

523 reduction during the feast phase was observed. Specifically, for the Enrichment reactor

524 characterization on operational day 144 (Fig. 5b), both $\mathrm{NO}_{2}$ and $\mathrm{NO}_{3}$ were reduced to nearly zero

525 after approximately $6 \mathrm{hrs}$, with a nominal increase in $\mathrm{NO}_{2}$ initially during the low-DO feast

526 period. Similar $\mathrm{NO}_{3}$ patterns were observed for the Enrichment reactor characterization on

527 operational day 190, indicating that $\mathrm{NO}_{3}$ was used as a terminal electron acceptor to support

528 heterotrophic respirative metabolic activities; conversely, autotrophic $\mathrm{NO}_{2}$ production was

529 realized over the same time period. In contrast to observations for the highest aerated reactor

530 (AE-20), these results indicate that residual $\mathrm{NO}_{3}$ from the preceding operational cycle was used 
531 by the MMC to sustain a VFA feast (and PHA synthesis), while the aeration state concurrently

532 supported partial nitritation. Results align with recent work on the same substrate (Hanson et al.

533 2016), as well as PHA production on sugar factory wastewater (Anterrieu et al. 2014).

534 qPCR was applied to quantify the relative NOB and AOB populations and better

535 understand the bulk solution observations. Regarding the NOB population, the relative fractional

536 abundance of Nitrobacter spp. (which exhibit a low affinity for $\mathrm{NO}_{2}$ and oxygen) for Enrichment

537 reactors AE-4, $-8,-12$ - and -20 was $1.8 \pm 1.0 \%, 2.6 \pm 2.2 \%, 3.5 \pm 3.5 \%$, and $5.9 \pm 4.8 \%$, respectively.

538 Relative fractions align with the imposed aeration state, as Nitrobacter spp. predominate at

539 elevated residual DO concentrations (i.e., highest for AE-20, lowest for AE-4). In contrast,

540 negligible Nitrospira were detected in any of the Enrichment reactors; Nitrospira typically

541 predominate at lower DO concentrations than observed herein. Regarding AOBs, relative

542 concentrations for Enrichment reactors AE-4, -8, -12- and -20 were $0.28 \pm 0.40 \%, 0.95 \pm 1.3 \%$,

$5430.81 \pm 1.7 \%$, and $0.43 \pm 0.81 \%$, respectively. AOBs exhibit a lower specific growth rate than NOBs

544 and thus would be present in lower concentrations (relative to NOBs). Collectively, these

545 molecular insights affirm the observed bulk solution nitrogen observations $\left(\mathrm{NH}_{4} \rightarrow \mathrm{NO}_{2} \rightarrow \mathrm{NO}_{3}\right)$

546 and also demonstrate the potential to enrich for flanking microbial populations that can generate

547 alternative electron acceptors to maintain feast-famine PHA production.

548 As a final observation regarding the observed nitrogen cycling, a secondary benefit arises

549 beyond supporting feast-famine PHA synthesis at a reduced aeration rate. U.S. dairies are

550 increasingly facing nitrogen-based environmental challenges. Thus, concurrent nitrogen

551 treatment would be a significant benefit in addition to commodity PHA production.

553 microbial composition of the MMC in the Enrichment reactors, on three operational days (29, 
55452 , and 271) amplicons of the V1-V3 region of the 16S rRNA gene for Eubacteria were

555 sequenced and assigned to phylotypes. The total number of joined sequences obtained are

556 summarized in Table S1. The number of reads ranged from 29 583-120 133, and sequencing

557 coverage depth was considered sufficient for all samples based on rarefaction analysis (Fig. S1),

558 although there was one lesser quality data set (MMC from reactor AE-12 on operational day 271;

559 Fig. S1c). While MMC in reactors AE-8 and -20 appeared to be somewhat more diverse, all four

560 Enrichment reactors generally exhibited similar complexity (average Shannon index of 3.74-

561 4.18; range of 3.59-4.41; Table S1). The Shannon index was selected for its value as a general

562 complexity measure considering both richness and evenness (Hill et al. 2003).

A comprehensive summary of the eubacterial-based MMC composition from the domain to

564 genus level for each Enrichment reactor is presented in Figs. S8-S23 (see also Tables S8-S37);

565 Fig. 6 presents a summary of the respective MMC's on operational day 271. Focusing on the

566 genera level (Table 5), Meganema was the most common and highly abundant genus observed

567 across all samples, ranging from 4.95-32.8\%; moreover, Meganema was the only genus present

568 at $>10 \%$ in all Enrichment reactors on operational days 29 and 52. However, on operational day

569271 Meganema decreased to $4.95-6.93 \%$ of the population, while Zoogloea was the only genus

$570>10 \%$ (and only in reactor AE-12). Though Meganema has been observed in other ADF

571 enrichment SBRs wherein PHA was synthesized in excess (Hanson et al. 2016, Majone et al.

572 2006), including with fermented dairy manure as a substrate (Hanson et al. 2016), the high

573 abundance of Meganema was in contrast to other ADF studies that reported dominant genera

574 including Thauera (Albuquerque et al. 2013, Carvalho et al. 2014, Lemos et al. 2008), Azoarcus

575 (Bengtsson et al. 2010a, Bengtsson et al. 2010b, Waller et al. 2012), or Plasticicumulans (Jiang

576 et al. 2011, Jiang et al. 2012, Marang et al. 2013). Additionally, most of the genera identified in 
577 this study present at >1\% (e.g., Leadbetterella, Haliscomenobacter, Aquabacterium, Luteimonas,

578 Hydrogenophaga, Thermomonas, Mycobacterium, Filomicrobium, Dokdonella, Hylemonella,

579 Pseudofulvimonas, Mesorhizobium, Hyphomicrobium, Parvibaculum, Brevundimonas,

580 Novosphingobium, Sphingomonas, Altererythrobacter, Xanthobacter, Gp6, and Gp4) have not

581 been reported in other ADF investigations; Amaricoccus and Paracoccus have only been

582 nominally present in ADF systems (Lemos et al. 2008, Queirós et al. 2014). Compared with

583 studies using synthetic medium (Albuquerque et al. 2013, Dionisi et al. 2006, Lemos et al. 2008,

584 Villano et al. 2010), real wastewater-based ADF operations appear to enrich for a much more

585 diverse population of potential PHA producing microorganisms. Indeed, the number of taxa

586 identified in this study was higher compared to these previous studies; moreover, the microbial

587 diversity (based on the Shannon index) was markedly higher than reported for an ADF

588 enrichment SBR cultivated on fermented molasses (Shannon index $\leq 1.0$ ) (Carvalho et al. 2014).

589 High-throughput DNA sequencing (a more sophisticated method as compared to FISH or DGGE

590 DNA band sequencing) may explain the more diverse MMC observed in this study, but other

591 possible explanations for the dissimilarity in microbial composition include differing inoculum

592 sources, feedstock composition, and applied operating conditions.

593 Independent of the differences in microbial composition across this study and also relative

594 to others, the feast-famine response at a bulk solution-level was observed in the Enrichment

595 reactors, indicating that the microbial composition may not be the strongest determinant in

596 eliciting a feast-famine response. Rather, the functional capabilities of the taxa present may be

597 more pertinent. To explore the functional potential associated with the microbial composition,

598 the major genera (Table 5) during the sampling events were further scrutinized. For the MiSeq

599 analysis, phylotypes were not assigned at the species level, meaning that the associated 
600 functional capabilities could not be established with absolute certainty. Thus, PHA synthesis

601 potential of the major genera was determined by i) comprehensive literature search of available

602 databases, ii) the genetic potential as established within the Uniprot database

603 (http://www.uniprot.org/) and the ribosomal database project (RDP), or iii) consideration of a

604 subordinate taxa (and their associated physiology) as a surrogate (e.g., as identified through the

605 MiDAS database (McIlroy et al. 2015). The potential to be capable of PHA synthesis was

606 established if a peer-reviewed study identified the genera or species as so capable, or if a mapped

607 genome was shown to include PHA related genes (e.g., PHA synthase, PHA depolymerase, PhaR

608 (Maehara et al. 2002), etc.). Genera exhibiting potential as PHA producers in the respective

609 Enrichment reactors are highlighted in Table 5 (bold and underlined). As shown, 21.5-49.9\% of

610 the MMC for genera present at $>1 \%$ have the potential to synthesize PHA. Specifically

611 pertaining to the most dominant genera observed (Meganema), its capabilities may be evaluated

612 by considering the only species currently in the RDP database, namely $M$. perideroedes, as a

613 proxy. $M$. perideroedes is a filamentous organism that was identified in activated sludge

614 (Thomsen et al. 2006) and was shown to exhibit rapid substrate uptake (including acetate,

615 propionate, and butyrate) using various electron acceptors (oxygen, nitrite, and nitrate) in

616 addition to a high PHA-accumulation capacity (Kragelund et al. 2005, Thomsen et al. 2006).

617 These metabolic characteristics of $M$. perideroedes, coupled with the observation of more than

618 half of the major phylotypes identified having at least one member known to possess a subset of

619 VFA assimilation or PHA synthesis, aligned with the observed bulk solution changes in the

620 Enrichment reactors; the denitrification potential also aligned well with Enrichment reactor

621 performance. Thauera, another prominent PHA-producing microbe that was observed in nearly

622 all MMC at $>1 \%$, is also capable of denitrification (Morgan-Sagastume et al. 2008). The 
623 presence of denitrifying PHA-producing microorganisms fits well with the observed nitrogen

624 cycling, particularly in Enrichment reactors AE-4, -8, and -12. As a final note, the MMC

625 contained filamentous bacteria (e.g., Meganema, Haliscomenobacter); with no settling phase

626 incorporated into the SBR operation, such an outcome is not surprising.

627 Finally, considering MMC diversity as related to PHA production potential, some of the

628 highest maximum PHA contents achieved (75-80\% PHA (VSS basis) for waste feedstocks

629 (Albuquerque et al. 2010, Carvalho et al. 2014) and upwards of 90\% PHB (TSS basis) for

630 synthetic substrates (Jiang et al. 2011, Johnson et al. 2009)) were achieved with MMC

631 enrichments with low microbial diversity. Results from Hanson et al. (2016) for PHA production

632 on sterilized dairy manure fermenter liquor further indicate a similar relationship. Thus, it could

633 be suggested that lower microbial diversity in enriched MMCs may be favorable for fed-batch

634 PHA production, particularly when increased microbial diversity is associated with more non-

635 PHA producing bacteria - an observation other investigations have also made (Jiang et al. 2012,

636 Queirós et al. 2014, Tamis et al. 2014). However, a similar analysis on this study's data was not

637 so conclusive; no relationship was observed between MMC diversity and \%PHA or PHA yield

638 (data not shown). Applying a Bray-Curtis similarity analysis to the respective MMC, no

639 discernable clustering by reactor was observed (Fig. S7 and Table S7). The respective MMC did

640 generally cluster by operational day, but significant dissimilarity existed. MMC in AE-4 and -8

641 exhibited the most similarity ( $80 \%$ on operational day $271 ; 76 \%$ on operational day 29 ), although

642 similarity across reactors generally was less than 60\%. Collectively, the microbial ecology

643 results suggest functional redundancy across diverse MMC is realized in ADF-operated reactors

644 fed a complex (waste-based) substrate.

$645 \quad 4 . \quad$ Conclusions 
Investigations were undertaken to evaluate the effect of aeration (controlled using the

647 oxygen mass transfer coefficient, $\mathrm{k}_{\mathrm{L}}$ a) on feast-famine PHA synthesis by mixed microbial

648 consortia fed VFA-rich fermented dairy manure; applied $\mathrm{k}_{\mathrm{L}}$ a values were 4, 8, 12, and $20 \mathrm{hr}^{-1}$,

649 and residual dissolved oxygen was measured as a response parameter. Assessment of PHA

650 Enrichment reactors revealed no statistical effect from reduced aeration on rates of carbon uptake

651 and storage. Indeed, regardless of the aeration state, all MMC exhibited a feast-famine response

652 based on observed carbon cycling. While a typical feast-famine dissolved oxygen (DO) pattern

653 was observed at maximum aeration, less resolution was observed at decreasing aeration rates,

654 suggesting that DO may not be an optimal process monitoring parameter. Moreover, lower

655 aeration states did appear to impair excess accumulation of PHA by MMC in the Production

656 reactors. At lower aeration states, nitrogen cycling revealed that alternative electron acceptors

$657\left(\mathrm{NO}_{2}, \mathrm{NO}_{3}\right)$ sustained feast-famine PHA synthesis mechanisms; molecular investigations

658 targeting AOBs and NOBs supported these observations. Next-generation sequencing analysis of

659 the MMC revealed very diverse populations across the applied aeration states. Numerous and

660 diverse genera observed in the respective Enrichment reactor MMCs are putative PHA

661 producers, suggesting functional redundancy. Ultimately, results demonstrate that aeration can

662 be controlled in waste-based ADF systems to sustain PHA production potential, while enriching

663 for a diverse MMC that exhibits potential functional redundancy. Reduced aeration could

664 enhance cost competitiveness of waste-based PHA production, with potential further benefits

665 associated with nitrogen treatment.

666 Acknowledgements

667

The material presented and discussed herein is based upon work supported by i) the U.S.

668

Department of Agriculture under Grant Number NIFA\#2012-68002-19952, ii) the National 
669 Science Foundation under Grant Number CBET-1235885, iii) the Idaho Dairymen's Association, 670 and iv) an Institutional Development Award (IDeA) from the National Institute of General

671 Medical Sciences of the National Institutes of Health under Grant Number P20GM103408. Any

672 opinions, findings, and conclusions or recommendations expressed in this material are those of

673 the authors and do not necessarily reflect the views of the funding agencies.

674 The authors greatly appreciate the efforts of Nicholas M. Guho, who prepared and

675 executed workflows to evaluate the MiSeq results and generate the associated tables and figures.

676 


\section{References.}

Akiyama, M., Tsuge, T. and Doi, Y. (2003) Environmental life cycle comparison of polyhydroxyalkanoates produced from renewable carbon resources by bacterial fermentation. Polym. Degrad. Stabil. 80(1), 183-194.

Albuquerque, M.G.E., Torres, C.A.V. and Reis, M.A.M. (2010) Polyhydroxyalkanoate (PHA) production by a mixed microbial culture using sugar molasses: Effect of the influent substrate concentration on culture selection. Water Res. 44(11), 3419-3433.

Albuquerque, M.G.E., Carvalho, G., Kragelund, C., Silva, A.F., Crespo, M.T.B., Reis, M.A.M. and Nielsen, P.H. (2013) Link between microbial composition and carbon substrateuptake preferences in a PHA-storing community. ISME J 7(1), 1-12.

Anterrieu, S., Quadri, L., Geurkink, B., Dinkla, I., Bengtsson, S., Arcos-Hernandez, M., Alexandersson, T., Morgan-Sagastume, F., Karlsson, A., Hjort, M., Karabegovic, L., Magnusson, P., Johansson, P., Christensson, M. and Werker, A. (2014) Integration of biopolymer production with process water treatment at a sugar factory. New Biotechnol. 31(4), 308-323.

APHA, AWWA and WEF (2012) Standard methods for the examination of water and wastewater, AWWA.

Bengtsson, S., Pisco, A.R., Johansson, P., Lemos, P.C. and Reis, M.A.M. (2010a) Molecular weight and thermal properties of polyhydroxyalkanoates produced from fermented sugar molasses by open mixed cultures. J Biotechnol 147(3-4), 172-179.

Bengtsson, S., Pisco, A.R., Reis, M.A.M. and Lemos, P.C. (2010b) Production of polyhydroxyalkanoates from fermented sugar cane molasses by a mixed culture enriched in glycogen accumulating organisms. J Biotechnol 145(3), 253-263.

Beun, J.J., Dircks, K., Van Loosdrecht, M.C.M. and Heijnen, J.J. (2002) Poly-B-hydroxybutyrate metabolism in dynamically fed mixed microbial cultures. Water Res. 36(5), 1167-1180.

Braunegg, G., Sonnleitner, B. and Lafferty, R.M. (1978) A rapid gas chromatographic method for the determination of poly- $\beta$-hydroxybutyric acid in microbial biomass. Eur. J. Appl. Microbiol. 6, 29-37.

Braunegg, G., Lefebvre, G. and Genser, K.F. (1998) Polyhydroxyalkanoates, biopolyesters from renewable resources: Physiological and engineering aspects. J. Biotechnol. 65(2-3), 127161.

Carvalho, G., Oehmen, A., Albuquerque, M.G.E. and Reis, M.A.M. (2014) The relationship between mixed microbial culture composition and PHA production performance from fermented molasses. New Biotechnol. 31(4), 257-263.

Chen, G.-Q. and Patel, M.K. (2012) Plastics Derived from Biological Sources: Present and Future: A Technical and Environmental Review. Chem. Rev. 112(4), 2082-2099.

Clesceri LS, G.A., Eaton AD (1998) Standard Methods for Examination of Water and Wastewater, American Public Health Association, Washington D.C.

Coats, E.R., Searcy, E., Feris, K., Shrestha, D., McDonald, A.G., Briones, A., Magnusson, T. and Prior, M. (2013) An integrated 2-stage anaerobic digestion and biofuel production process to reduce life cycle GHG emissions from U.S. dairies. BioFPR 7(4), 459-473.

Dias, J.M.L., Serafim, L.S., Lemos, P.C., Reis, M.A.M. and Oliveira, R. (2005) Mathematical modelling of a mixed culture cultivation process for the production of polyhydroxybutyrate. Biotechnol. Bioeng. 92(2), 209-222.

Dias, J.M.L., Lemos, P.C., Serafim, L.S., Oliveira, C., Eiroa, M., Albuquerque, M.G.E., Ramos, A.M., Oliveira, R. and Reis, M.A. (2006) Recent advances in polyhydroxyalkanoate 
production by mixed aerobic cultures: from the substrate to the final product. Macromol. Biosci. 6, 885-906.

Dionisi, D., Majone, M., Tandoi, V. and Beccari, M. (2001) Sequencing batch reactor: Influenct of periodic operation on performance of activated sludges in biological wastewater treatment. Ind Eng Chem Res 40, 5110-5119.

Dionisi, D., Renzi, V., Majone, M., Beccari, M. and Ramadori, R. (2004) Storage of substrate mixtures by activated sludges under dynamic conditions in anoxic or aerobic environments. Water Res. 38(8), 2196-2206.

Dionisi, D., Majone, M., Vallini, G., Di Gregorio, S. and Beccari, M. (2006) Effect of the applied organic load rate on biodegradable polymer production by mixed microbial cultures in a sequencing batch reactor. Biotechnol. Bioeng. 93(1), 76-88.

Dionisi, D., Majone, M., Vallini, G., DiGregorio, S. and Beccari, M. (2007) Effect of the length of the cycle on biodegradable polymer production and microbial community selection in a sequencing batch reactor. Biotechnol Prog 23(5), 1064-1073.

Fernández-Dacosta, C., Posada, J.A., Kleerebezem, R., Cuellar, M.C. and Ramirez, A. (2015) Microbial community-based polyhydroxyalkanoates (PHAs) production from wastewater: Techno-economic analysis and ex-ante environmental assessment. Bioresour. Technol. 185, 368-377.

Garcia-Ochoa, F. and Gomez, E. (2009) Bioreactor scale-up and oxygen transfer rate in microbial processes: An overview. Biotechnol. Adv. 27(2), 153-176.

Gurieff, N. and Lant, P. (2007) Comparative life cycle assessment and financial analysis of mixed culture polyhydroxyalkanoate production. Bioresour. Technol. 98(17), 3393-3403.

Hanson, A.J., Guho, N.M., Paszczynski, A.J. and Coats, E.R. (2016) Community proteomics provides functional insight into polyhydroxyalkanoate production by a mixed microbial culture cultivated on fermented dairy manure. Appl. Biochem. Biotechnol., 1-20.

Hill, T.C.J., Walsh, K.A., Harris, J.A. and Moffett, B.F. (2003) Using ecological diversity measures with bacterial communities. FEMS Microbiol Ecol 43(1), 1-11.

Jiang, Y., Marang, L., Kleerebezem, R., Muyzer, G. and van Loosdrecht, M.C.M. (2011) Polyhydroxybutyrate production from lactate using a mixed microbial culture. Biotechnol Bioeng 108(9), 2022-2035.

Jiang, Y., Marang, L., Tamis, J., van Loosdrecht, M.C.M., Dijkman, H. and Kleerebezem, R. (2012) Waste to resource: Converting paper mill wastewater to bioplastic. Water Res. 46(17), 5517-5530.

Johnson, K., Jiang, Y., Kleerebezem, R., Muyzer, G. and van Loosdrecht, M.C.M. (2009) Enrichment of a Mixed Bacterial Culture with a High Polyhydroxyalkanoate Storage Capacity. Biomacromolecules 10(4), 670-676.

Kragelund, C., Nielsen, J.L., Thomsen, T.R. and Nielsen, P.H. (2005) Ecophysiology of the filamentous Alphaproteobaeterium Meganema perideroedes in activated sludge. FEMS Microbiol Ecol 54(1), 111-122.

Leininger, S., Urich, T., Schloter, M., Schwark, L., Qi, J., Nicol, G.W., Prosser, J.I., Schuster, S.C. and Schleper, C. (2006) Archaea predominate among ammonia-oxidizing prokaryotes in soils. Nature 442(7104), 806-809.

Lemoigne, M. (1926) Produits de deshydration et de polymerisation de l'acide b-oxobutyrique. Bull. Soc. Chem. Biol. (Paris) 8, 770-782.

Lemos, P.C., Levantesi, C., Serafim, L.S., Rossetti, S., Reis, M.A.M. and Tandoi, V. (2008) Microbial characterisation of polyhydroxyalkanoates storing populations selected under 
801

802

803

804

805

806

807

808

809

810

811

812

different operating conditions using a cell-sorting RT-PCR approach. Appl. Microbiol. Biotechnol. 78(2), 351-360.

Luzier, W.D. (1992) Materials derived from biomass/biodegradable materials. PNAS 89, 839842.

Madison, L.L. and Huisman, G.W. (1999) Metabolic engineering of poly (3-hydroxyalkanoates): from DNA to plastic. Microbiol. Mol. Biol. Rev. 63(1), 21-53.

Maehara, A., Taguchi, S., Nishiyama, T., Yamane, T. and Doi, Y. (2002) A repressor protein, PhaR, regulates polyhydroxyalkanoate (PHA) synthesis via its direct interaction with PHA. J. Bacteriol. 184(14), 3992-4002.

Magoč, T. and Salzberg, S.L. (2011) FLASH: fast length adjustment of short reads to improve genome assemblies. Bioinformatics 27(21), 2957-2963.

Majone, M., Massanisso, P., Carucci, A., Lindrea, K. and Tandoi, V. (1996) Influence of storage on kinetic selection to control aerobic filamentous bulking. Water Sci. Technol. 34(5-6), 223-232.

Majone, M., Beccari, M., Di Gregorio, S., Dionisi, D. and Vallini, G. (2006) Enrichment of activated sludge in a sequencing batch reactor for polyhydroxyalkanoate production. Water Sci Technol 54(1), 119-128.

Marang, L., Jiang, Y., van Loosdrecht, M.C.M. and Kleerebezem, R. (2013) Butyrate as preferred substrate for polyhydroxybutyrate production. Bioresource Technol 142, 232239.

McIlroy, S.J., Saunders, A.M., Albertsen, M., Nierychlo, M., McIlroy, B., Hansen, A.A., Karst, S.M., Nielsen, J.L. and Nielsen, P.H. (2015) MiDAS: the field guide to the microbes of activated sludge. Database 2015.

Moralejo-Gárate, H., Kleerebezem, R., Mosquera-Corral, A. and van Loosdrecht, M.C.M. (2013) Impact of oxygen limitation on glycerol-based biopolymer production by bacterial enrichments. Water Res. 47(3), 1209-1217.

Morgan-Sagastume, F., Nielsen, J.L. and Nielsen, P.H. (2008) Substrate-dependent denitrification of abundant probe-defined denitrifying bacteria in activated sludge. FEMS Microbiol. Ecol. 66(2), 447-461.

Muyzer, G., de Waal, E.C. and Uitterlinden, A.G. (1993) Profiling of complex microbial populations by denaturing gradient gel electrophoresis analysis of polymerase chain reaction-amplified genes coding for 16S rRNA. Appl. Environ. Microbiol. 59(3), 695700.

Padermshoke, A., Katsumotoc, Y., Satoa, H., Ekgasitb, S., Nodad, I. and Ozakia, Y. (2004) Surface melting and crystallization behavior of polyhydroxyalkanoates studied by attenuated total reflection infrared spectroscopy. Polymer 45, 6547-6554.

Passero, M.L., Cragin, B., Hall, A.R., Staley, N., Coats, E.R., McDonald, A.G. and Feris, K. (2014) Ultraviolet radiation pre-treatment modifies dairy wastewater, improving its utility as a medium for algal cultivation. Algal Res. 6, Part A(0), 98-110.

Queirós, D., Rossetti, S. and Serafim, L.S. (2014) PHA production by mixed cultures: A way to valorize wastes from pulp industry. Bioresour. Technol. 157, 197-205.

Ramakers, C., Ruijter, J.M., Deprez, R.H.L. and Moorman, A.F.M. (2003) Assumption-free analysis of quantitative real-time polymerase chain reaction (PCR) data. Neuroscience Letters 339(1), 62-66. 
Reis, M.A.M., Serafim, L.S., Lemos, P.C., Ramos, A.M., Aguiar, F.R. and van Loosdrecht, M.C.M. (2003) Production of polyhydroxyalkanoates by mixed microbial cultures. Bioprocess Biosyst. Eng. 25, 377-385.

Rotthauwe, J.H., Witzel, K.P. and Liesack, W. (1997) The ammonia monooxygenase structural gene amoA as a functional marker: molecular fine-scale analysis of natural ammoniaoxidizing populations. Appl. Environ. Microbiol. 63(12), 4704-4712.

Rudnik, E. (2008) Compostable Polymer Materials, Elsevier, Oxford, UK.

Serafim, L.S., Lemos, P.C., Oliveira, R. and Reis, M.A.M. (2004) Optimization of polyhydroxybutyrate production by mixed microbial cultures submitted to aerobic dynamic feeding conditions. Biotechnol. Bioeng. 87(2), 145-160.

Serafim, L.S., Lemos, P.C., Albuquerque, M.G.E. and Reis, M.A.M. (2008) Strategies for PHA production by mixed cultures and renewable waste materials. Appl. Microbiol. Biotechnol. 81, 615-628.

Shen, J., Song, N., Williams, C.J., Brown, C.J., Yan, Z., Xu, C. and Forney, L.J. (2016) Effects of low dose estrogen therapy on the vaginal microbiomes of women with atrophic vaginitis. Scientific Reports 6, 24380.

Shen, L., Worrell, E. and Patel, M. (2010) Present and future development in plastics from biomass. BioFPR 4, 25-40.

Stowe, E.J., Coats, E.R. and Brinkman, C.K. (2015) Dairy Manure Resource Recovery utilizing Two-stage Anaerobic Digestion - Implications of Solids Fractionation. Bioresour. Technol. 198, 237-245.

Tamis, J., Lužkov, K., Jiang, Y., van Loosdrecht, M.C. and Kleerebezem, R. (2014) Enrichment of Plasticicumulans acidivorans at pilot-scale for PHA production on industrial wastewater. J Biotechnol 192, 161-169.

Tchobanoglous, G., Stensel, H.D., Tsuchihashi, R. and Burton, F. (2014) Wastewater engineering: Treatment and resource recovery, Metcalf \& Eddy/AECOM, New York, NY.

Third, K.A., Burnett, N. and Cord-Ruwisch, R. (2003a) Simultaneous nitrification and denitrification using stored substrate (PHB) as the electron donor in an SBR. Biotechnol. Bioeng. 83(6), 706-720.

Third, K.A., Newland, M. and Cord-Ruwisch, R. (2003b) The effect of dissolved oxygen on PHB accumulation in activated sludge cultures. Biotechnol. Bioeng. 82(2), 238-250.

Third, K.A., Sepramaniam, S., Tonkovic, Z., Newland, M. and Cord-Ruwisch, R. (2004) Optimisation of storage driven denitrification by using on-line specific oxygen uptake rate monitoring during SND in a SBR. Water Sci. Technol. 50(10), 171-180.

Thomsen, T.R., Blackall, L.L., de Muro, M.A., Nielsen, J.L. and Nielsen, P.H. (2006) Meganema perideroedes gen. nov., sp nov., a filamentous alphaproteobacterium from activated sludge. Int J Syst Evol Micr 56, 1865-1868.

Valentino, F., Karabegovic, L., Majone, M., Morgan-Sagastume, F. and Werker, A. (2015) Polyhydroxyalkanoate (PHA) storage within a mixed-culture biomass with simultaneous growth as a function of accumulation substrate nitrogen and phosphorus levels. Water Res. 77, 49-63.

van Loosdrecht, M.C.M., Pot, M.A. and Heijnen, J.J. (1997) Importance of bacterial storage polymers in bioprocesses. Water Sci. Technol. 35(1), 41-47.

Van Wegen, R.J., Ling, Y. and Middelberg, A.P.J. (1998) Industrial production of PHAs using Escherichia coli: An economic analysis. Trans. IChemE 76(3), 417-426. 
Villano, M., Beccari, M., Dionisi, D., Lampis, S., Miccheli, A., Vallini, G. and Majone, M. (2010) Effect of $\mathrm{pH}$ on the production of bacterial polyhydroxyalkanoates by mixed cultures enriched under periodic feeding. Proc. Biochem. 45(5), 714-723.

Waller, J.L., Green, P.G. and Loge, F.J. (2012) Mixed-culture polyhydroxyalkanoate production from olive oil mill pomace. Bioresource Technol 120, 285-289.

Wang, Q., Garrity, G.M., Tiedje, J.M. and Cole, J.R. (2007) Naïve Bayesian classifier for rapid assignment of rRNA sequences into the New Bacterial Taxonomy. Appl Environ Microbiol 73(16), 5261-5267.

Winkler, M., Coats, E.R. and Brinkman, C.K. (2011) Advancing post-anoxic denitrification for biological nutrient removal. Water Res. 45(18), 6119-6130.

Yates, M.R. and Barlow, C.Y. (2013) Life cycle assessments of biodegradable, commercial biopolymers-A critical review. Resour. Conserv. Recy. 78, 54-66. 
873 Table 1 Experimental reactor operating conditions

874 Table 2 PHA synthesis performance summary in the Enrichment reactors. Results for PHA Yield, $\mathrm{PHA}_{\max }$, and \% $\mathrm{PHA}_{\max }$ represent values at maximum observed intracellular PHA concentration. Average \%HV represents the average HV content in the PHA throughout the feast phase and subsequent PHA utilization during the famine phase.

879 Table 3 Rates of VFA uptake and PHA synthesis in the Enrichment reactors ( $\mathrm{n}=5$ per 880 reactor) through the feast phase.

881

882

883

884

885

Table 4 Performance data for PHA production assessments using inocula from the four different aeration-state Enrichment reactors. Y represents the total VFAs consumed up to the maximum observed intracellular PHA concentration. $\% \mathrm{PHA}_{\max }$ represents the maximum observed intracellular concentration during the production assessment. Avg. HV\% represents the mol-basis HV content over the full PHA production assessment.

Table 5 Summary of Major (>10\%) and Minor (1-10\%) genera observed in the four PHA Enrichment reactors on operational days 29, 52, and 271. Genera underlined and in bold text all contain species that exhibit PHA production potential (fraction of the respective MMC shown in parentheses). 


\section{LIST OF FIGURES}

Figure 1 VFA and PHA profiles for the Enrichment reactors operated at oxygen mass transfer coefficients $\left(\mathrm{k}_{\mathrm{L}} \mathrm{a}\right)$ of $20 \mathrm{hr}^{-1}(\mathrm{a}), 12 \mathrm{hr}^{-1}(\mathrm{~b}), 8 \mathrm{hr}^{-1}(\mathrm{c})$, and $4 \mathrm{hr}^{-1}(\mathrm{~d})$. Note that PHA reserves at the end of the SBR cycle were depleted.

Figure 2 Residual dissolved oxygen profiles for the Enrichment reactors during the feast phase and into the famine phase for reactors operating at oxygen mass transfer coefficients (k $\left.\mathrm{k}_{\mathrm{L}} \mathrm{a}\right)$ of $20 \mathrm{hr}^{-1}$ (a), $12 \mathrm{hr}^{-1}(\mathrm{~b}), 8 \mathrm{hr}^{-1}$ (c), and $4 \mathrm{hr}^{-1}(\mathrm{~d})$.

Figure 3 Example PHA production data (stage 1-B) for biomass from the Enrichment reactors operated at oxygen mass transfer coefficients $\left(k_{L}\right.$ a) of $20 \mathrm{hr}^{-1}(a), 12 \mathrm{hr}^{-1}(b), 8 \mathrm{hr}^{-1}(\mathrm{c})$, and $4 \mathrm{hr}^{-1}$ (d). Operations were conducted to supply new substrate (VFA-rich fermenter liquor) to the biomass based on a sudden increase in dissolved oxygen.

Figure 4 Example PHA Production data (stage 2-B) for biomass from the Enrichment reactors operated at oxygen mass transfer coefficients $\left(\mathrm{k}_{\mathrm{L}} \mathrm{a}\right)$ of $20 \mathrm{hr}^{-1}$ (a), $12 \mathrm{hr}^{-1}(\mathrm{~b}), 8 \mathrm{hr}^{-1}(\mathrm{c})$, and $4 \mathrm{hr}^{-1}$ (d). Operations were conducted to supply new substrate (VFA-rich fermenter liquor) to the biomass every 30 minutes.

Figure 5 Nitrogen cycling in Enrichment reactors operated at oxygen mass transfer coefficients $\left(\mathrm{k}_{\mathrm{L}} \mathrm{a}\right)$ of $20 \mathrm{hr}^{-1}$ (a) and $4 \mathrm{hr}^{-1}$ (b). Note that the abscissa scale begins at -10 minutes to reflect nitrogen concentrations before the cycle operations commenced.

Figure 6 Relative abundance and taxonomic classification of the 16S rRNA gene sequencing results using the RDP for Enrichment reactors on operational day 271 and operated at oxygen mass transfer coefficients $\left(\mathrm{k}_{\mathrm{L}} \mathrm{a}\right)$ of $20 \mathrm{hr}^{-1}(\mathrm{a}), 12 \mathrm{hr}^{-1}(\mathrm{~b}), 8 \mathrm{hr}^{-1}(\mathrm{c})$, and $4 \mathrm{hr}^{-1}$ (d). The classified phylotypes are depicted in terms of the taxonomic hierarchy. Phylotypes which were not identified by the RDP or those whose identification at a specific taxonomic level was not statistically significant were aggregated, denoted "No 
ID”, and depicted in red. Identified phylotypes with less than $1 \%$ of the total relative abundance were aggregated, denoted "Minor", and depicted in gray. Phylotypes with at least $1 \%$ relative abundance are labeled. 
Table 1

\begin{tabular}{cccccc}
\hline Reactor & $\begin{array}{c}\text { Operating } \\
\text { Volume } \\
(\mathbf{L})\end{array}$ & $\begin{array}{c}\mathbf{k}_{\mathbf{L}} \mathbf{a} \\
\left(\mathbf{h r}^{-1}\right)\end{array}$ & $\begin{array}{c}\text { Air Flow } \\
\left(\mathbf{m L ~ m i n}^{-1}\right)\end{array}$ & $\begin{array}{c}\text { Avg. Flow } \\
\text { Reduction } \\
\text { from AE-20 }\end{array}$ & $\begin{array}{c}\text { SRT and } \\
\text { HRT (d) }\end{array}$ \\
\hline AE-4 & 1.2 & 4 & 15 & $97.5 \%$ & 4 \\
AE-8 & 1.2 & 8 & 80 & $87 \%$ & 4 \\
AE-12 & 1.2 & 12 & 245 & $59 \%$ & 4 \\
AE-20 & 1.2 & 20 & 600 & NA & 4 \\
\hline
\end{tabular}


Table 2

\begin{tabular}{|c|c|c|c|c|c|c|c|c|}
\hline \multirow[t]{2}{*}{$\begin{array}{c}\mathbf{k}_{\mathrm{L}} \mathbf{a} \\
\left(\mathbf{h r}^{-1}\right)\end{array}$} & \multirow[t]{2}{*}{$\begin{array}{c}\text { Operational } \\
\text { Day }\end{array}$} & \multicolumn{2}{|c|}{$\begin{array}{c}\text { PHA Yield } \\
(\text { CmmolPHA } \\
\left.(\text { CmmolVFA })^{-1}\right)\end{array}$} & \multicolumn{2}{|c|}{$\underset{\left(\text { Cmmol L }^{-1}\right)}{\text { PHA }_{\max }}$} & \multicolumn{2}{|c|}{ 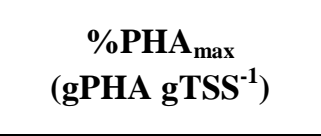 } & \multirow[t]{2}{*}{$\begin{array}{l}\text { Avg. HV\% } \\
\text { (mol basis) }\end{array}$} \\
\hline & & & avg. \pm SD & & avg. \pm SD & & avg. \pm SD & \\
\hline \multirow{5}{*}{4} & 29 & 0.36 & & 5.81 & & 7.32 & & \multirow{5}{*}{$\begin{array}{c}35.9 \pm 16.8 \\
(\mathrm{n}=32)\end{array}$} \\
\hline & 52 & 0.51 & & 8.98 & & 17.10 & & \\
\hline & 144 & 0.41 & $0.53 \pm 0.21$ & 3.44 & $7.31 \pm 2.61$ & 5.50 & $10.55 \pm 4.54$ & \\
\hline & 190 & 0.90 & & 9.68 & & 10.39 & & \\
\hline & 271 & 0.49 & & 8.61 & & 12.45 & & \\
\hline \multirow{5}{*}{8} & 29 & 0.46 & & 7.29 & & 10.25 & & \multirow{5}{*}{$\begin{array}{c}30.8 \pm 15.8 \\
(\mathrm{n}=36)\end{array}$} \\
\hline & 52 & 0.41 & & 7.02 & & 12.85 & & \\
\hline & 144 & 0.50 & $0.59 \pm 0.21$ & 4.28 & $7.79 \pm 2.48$ & 5.57 & $10.74 \pm 3.60$ & \\
\hline & 190 & 0.93 & & 10.07 & & 9.86 & & \\
\hline & 271 & 0.63 & & 10.28 & & 15.17 & & \\
\hline \multirow{5}{*}{12} & 29 & 0.27 & & 4.33 & & 4.41 & & \multirow{5}{*}{$\begin{array}{c}25.7 \pm 19.7 \\
(n=37)\end{array}$} \\
\hline & 52 & 0.54 & & 9.25 & & 12.60 & & \\
\hline & 144 & 1.16 & $0.70 \pm 0.34$ & 10.00 & $8.73 \pm 2.48$ & 11.24 & $10.90 \pm 4.05$ & \\
\hline & 190 & 0.92 & & 9.88 & & 10.81 & & \\
\hline & 271 & 0.63 & & 10.18 & & 15.44 & & \\
\hline \multirow{5}{*}{20} & 29 & 0.34 & & 5.34 & & 6.85 & & \multirow{5}{*}{$\begin{array}{c}38.0 \pm 15.9 \\
(\mathrm{n}=38)\end{array}$} \\
\hline & 52 & 0.42 & & 7.41 & & 12.50 & & \\
\hline & 144 & 1.30 & $0.75 \pm 0.39$ & 11.01 & $9.92 \pm 3.90$ & 18.27 & $14.12 \pm 6.17$ & \\
\hline & 190 & 0.95 & & 10.26 & & 10.65 & & \\
\hline & 271 & 0.76 & & 15.60 & & 22.33 & & \\
\hline
\end{tabular}


Table 3

\begin{tabular}{|c|c|c|c|c|}
\hline $\mathbf{k}_{\mathbf{L}} \mathbf{a}$ & $\begin{array}{c}\text { qVFA }_{\mathrm{VFA}} \\
\left(\mathrm{Cmmol} \mathrm{gVSS}^{-1} \mathrm{~min}^{-1}\right) \\
\end{array}$ & $\begin{array}{c}\mathbf{r}_{\mathrm{VFA}} \\
\left(\mathrm{Cmmol} \mathrm{L}^{-1} \mathrm{~min}^{-1}\right) \\
\end{array}$ & $\begin{array}{c}\text { qPHA } \\
\left(\mathrm{Cmmol} \mathrm{gVSS}^{-1} \mathrm{~min}^{-1}\right) \\
\end{array}$ & $\begin{array}{c}\mathbf{r}_{\text {PHA }} \\
\left(\mathrm{Cmmol} \mathrm{L}^{-1} \mathbf{m i n}^{-1}\right) \\
\end{array}$ \\
\hline 4 & $0.18 \pm 0.07$ & $0.22 \pm 0.09$ & $0.11 \pm 0.07$ & $0.13 \pm 0.09$ \\
\hline 8 & $0.20 \pm 0.09$ & $0.26 \pm 0.05$ & $0.10 \pm 0.04$ & $0.13 \pm 0.06$ \\
\hline 12 & $0.14 \pm 0.07$ & $0.20 \pm 0.08$ & $0.11 \pm 0.09$ & $0.14 \pm 0.10$ \\
\hline 20 & $0.26 \pm 0.13$ & $0.31 \pm 0.14$ & $0.19 \pm 0.10$ & $0.23 \pm 0.12$ \\
\hline
\end{tabular}


Table 4

\begin{tabular}{|c|c|c|c|c|c|c|}
\hline Reactor & $\begin{array}{c}\text { Production } \\
\text { Test (Op. } \\
\text { Day) }\end{array}$ & $\begin{array}{c}\text { Initial } \\
\text { MLSS } \\
\left(\mathbf{m g ~ L}^{-1}\right)\end{array}$ & $\begin{array}{c}\mathbf{Y} \\
\left(\text { CmmolPHA }^{-}\right. \\
\left.\text {CmmolVFA }^{-1}\right)\end{array}$ & $\begin{array}{l}\text { \%PHA }_{\max } \\
(\mathrm{mgPHA} \\
\left.\text { mgTSS }^{-1}\right)\end{array}$ & $\begin{array}{c}\mathrm{PHA}_{\max } \\
(\mathrm{mgPHA} \\
\left.\mathrm{mgVSS}^{-1}\right)\end{array}$ & $\begin{array}{l}\text { Avg. HV\% } \\
\text { (mol basis) }\end{array}$ \\
\hline \multirow{4}{*}{$\mathrm{AE}-4$} & 1-A (173) & 2590 & 0.78 & 40.5 & 49.4 & $27 \pm 5.2(n=16)$ \\
\hline & 1-B (238) & 2520 & 0.72 & 27.8 & 34.3 & $41 \pm 11(\mathrm{n}=18)$ \\
\hline & $2-A(263)$ & 1750 & 0.46 & 38.5 & 48.2 & $65 \pm 2.9(\mathrm{n}=6)$ \\
\hline & 2-B (271) & 1890 & 0.71 & 35.2 & 42.4 & $49 \pm 5.4(n=6)$ \\
\hline \multirow{4}{*}{ AE-8 } & $1-A(173)$ & 2740 & 0.97 & 47.0 & 59.6 & $25 \pm 5.4(\mathrm{n}=16)$ \\
\hline & 1-B (238) & 2490 & 0.77 & 18.3 & 22.5 & $43 \pm 10.0(n=16)$ \\
\hline & $2-A(263)$ & 1960 & 0.91 & 54.7 & 67.9 & $69 \pm 0.86(n=5)$ \\
\hline & 2-B (271) & 1780 & 0.68 & 29.7 & 35.3 & $44 \pm 3.8(n=6)$ \\
\hline \multirow{4}{*}{$\mathrm{AE}-12$} & $1-\mathrm{A}(173)$ & 2540 & 0.94 & 43.1 & 52.7 & $31 \pm 5.1(\mathrm{n}=17)$ \\
\hline & 1-B (238) & 2410 & 0.87 & 23.3 & 29.1 & $42 \pm 7.8(n=16)$ \\
\hline & $2-A(263)$ & 1790 & 1.12 & 70.9 & 90.7 & $73 \pm 0.6(n=6)$ \\
\hline & 2-B (271) & 1160 & 0.97 & 46.1 & 54.1 & $52 \pm 2.1(\mathrm{n}=6)$ \\
\hline \multirow{4}{*}{ AE-20 } & $1-A(173)$ & 1820 & 0.76 & 33.4 & 40.0 & $28 \pm 7.8(n=16)$ \\
\hline & 1-B (238) & 2460 & 0.93 & 28.3 & 35.5 & $42 \pm 4.8(\mathrm{n}=19)$ \\
\hline & $2-A(263)$ & 1940 & 0.97 & 62.6 & 78.3 & $73 \pm 0.52(n=5)$ \\
\hline & 2-B (271) & 1687 & 0.94 & 42.6 & 49.6 & $54 \pm 2.6(n=6)$ \\
\hline
\end{tabular}


Table 5

\begin{tabular}{|c|c|c|c|c|c|}
\hline \multicolumn{2}{|c|}{ Op. Day } & AE-4 & AE-8 & AE-12 & AE-20 \\
\hline \multirow[b]{2}{*}{29} & Major $(>10 \%)$ & Meganema $(27 \%)$ & Meganema $(16.1 \%)$ & Meganema $(19.1 \%)$ & Meganema (26\%) \\
\hline & $\begin{array}{l}\text { Minor } \\
(>1 \%)\end{array}$ & $\begin{array}{c}\frac{\text { Leadbetterella }(3.54 \%)}{\text { Luteimonas }(1.78 \%),}, \\
\frac{\text { Haliscomenobacter }(1.77 \%)}{\text { Planctomyces, }}, \\
\text { Thauera }(1.56 \%), \\
\frac{\text { Aquabacterium }(1.35 \%)}{\text { Gp4 }(1.32 \%),} \\
\frac{\text { Altererythrobacter }(1.2 \%),}{\text { Schlesneria }}\end{array}$ & 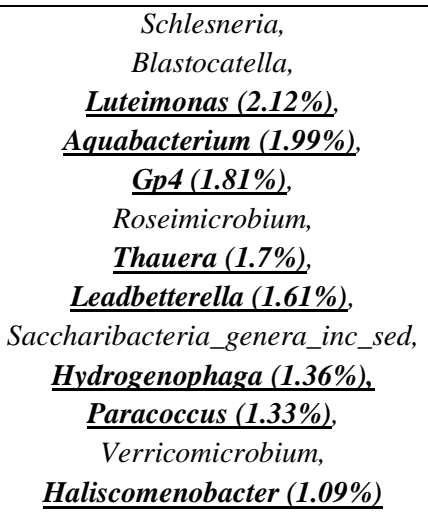 & 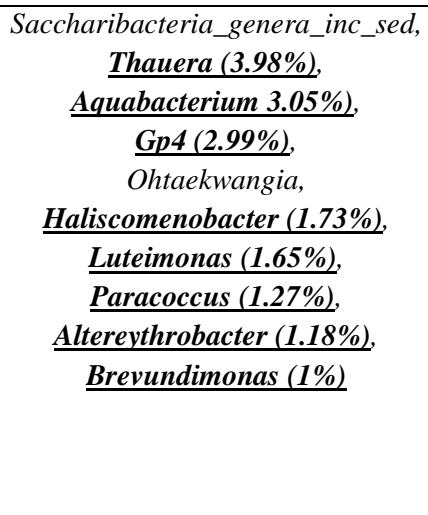 & $\begin{array}{c}\text { Ohtaekwangia, } \\
\text { Leadbetterella (6.16\%), } \\
\text { Planctomyces, } \\
\text { Pseudofulvimonas }(\mathbf{2 . 7 8 \% )}, \\
\text { Schlesneria, } \\
\text { Gp4(1.82\%)}, \\
\text { Saccharibacteria_genera_inc_sed, } \\
\text { Spartobacteria_genera_inc_sed, } \\
\text { Paracoccus }(\mathbf{1 . 1 8 \% )}, \\
\text { Armatimondadetes_gp5 }\end{array}$ \\
\hline \multirow[b]{2}{*}{52} & Major (>10\%) & Meganema $(11.4 \%)$ & Meganema $(17.7 \%)$ & Meganema $(32.8 \%)$ & Meganema $(15.8 \%)$ \\
\hline & $\begin{array}{l}\text { Minor } \\
(>1 \%)\end{array}$ & $\begin{array}{c}\text { Zoogloea (6.54\%), } \\
\text { Parcubacteria_genera_inc_sed, } \\
\text { Leadbetterella (3.62\%), } \\
\frac{\text { Luteimonas }(3.58 \%)}{\text { Nannocystis, }} \\
\text { Pedobacter, } \\
\text { Thauera }(\mathbf{1 . 8 6 \% )}, \\
\text { Ohtaekwangia, } \\
\text { Blastocatella, } \\
\text { Ilumatobacter, } \\
\text { Flavobacterium, } \\
\text { Proteiniphilum, } \\
\text { Hydrogenophaga }(\mathbf{1 . 0 4 \% )}\end{array}$ & 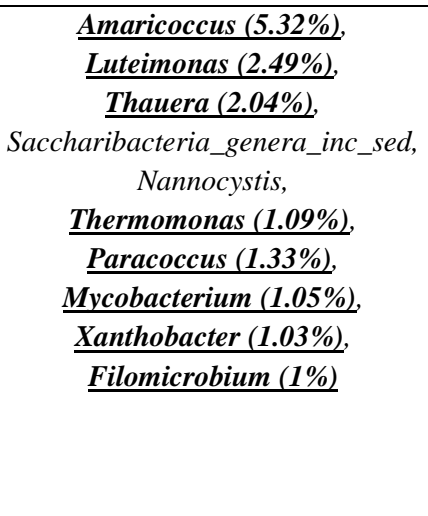 & 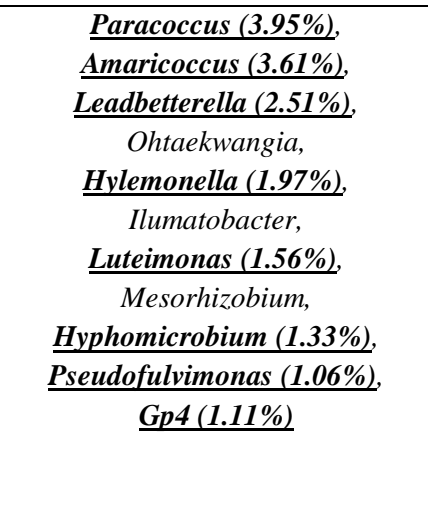 & 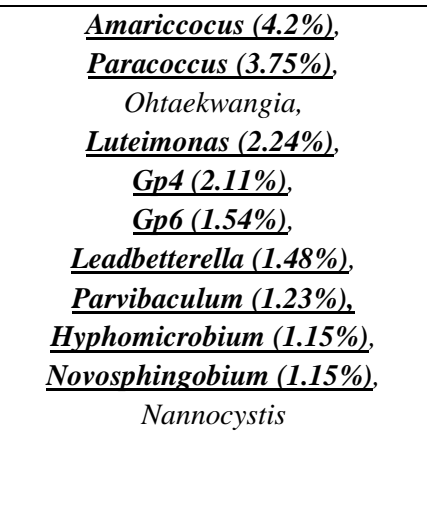 \\
\hline \multirow[b]{2}{*}{271} & Major $(>10 \%)$ & -- & -- & Zoogloea (24.3\%) & -- \\
\hline & $\begin{array}{l}\text { Minor } \\
(>1 \%)\end{array}$ & $\begin{array}{c}\frac{\text { Zoogloea }(9.9 \%)}{\text { Thauera }(7.87 \%)}, \\
\text { Meganema }(4.95 \%), \\
\text { Proteiniphilum, } \\
\text { Bacteroides, } \\
\text { Sphaerochaeta }\end{array}$ & $\begin{array}{l}\text { Thauera }(9.61 \%) \\
\frac{\text { Meganema }(6.93 \%)}{\text { Zoogloea }(5.45 \%)}, \\
\text { Proteiniphilum, } \\
\frac{\text { Dokdonella }(1.55 \%)}{\text { Bacteroides }}\end{array}$ & $\begin{array}{c}\text { Meganema }(6.92 \%), \\
\text { Bacteroides, } \\
\text { Spaerochaeta, } \\
\text { Proteiniphilum, } \\
\text { Thauera }(\mathbf{1 . 1 6 \% )}, \\
\text { Haliscomenobacter }(\mathbf{1 . 1 2 \% )} \\
\end{array}$ & $\begin{array}{c}\frac{\text { Meganema }(5.68 \%)}{\text { Gp4 }(4.81 \%),} \\
\text { Haliscomenobacter }(3.58 \%), \\
\text { Proteiniphilum, } \\
\text { Parcubacteria_genera_inc_sed, } \\
\frac{\text { Amaricoccus }(2.68 \%),}{\text { Thauera }(2.31 \%)} \\
\text { Oscillibacter, } \\
\text { Hallella, } \\
\text { Prosthecobacter, } \\
\text { Leadbetterella }(1.31 \%), \\
\text { Sphingomonas }(1.13 \%)\end{array}$ \\
\hline
\end{tabular}


Figure 1a

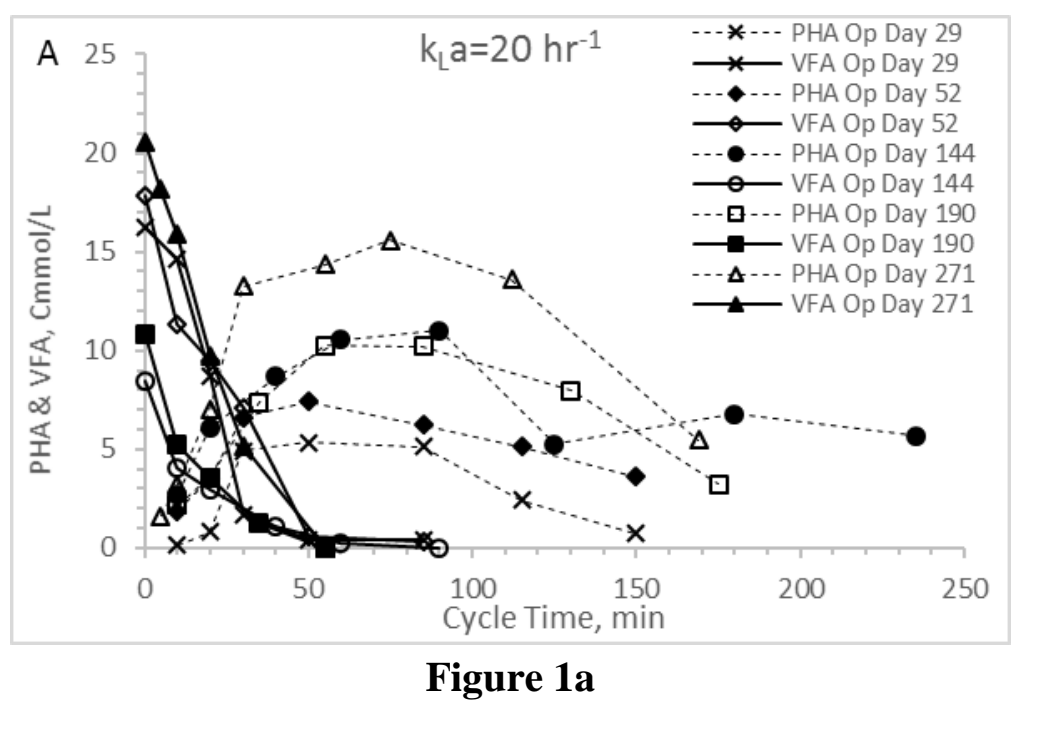

Figure 1a

Figure 1a

Figure 1a

. 
Figure 1b

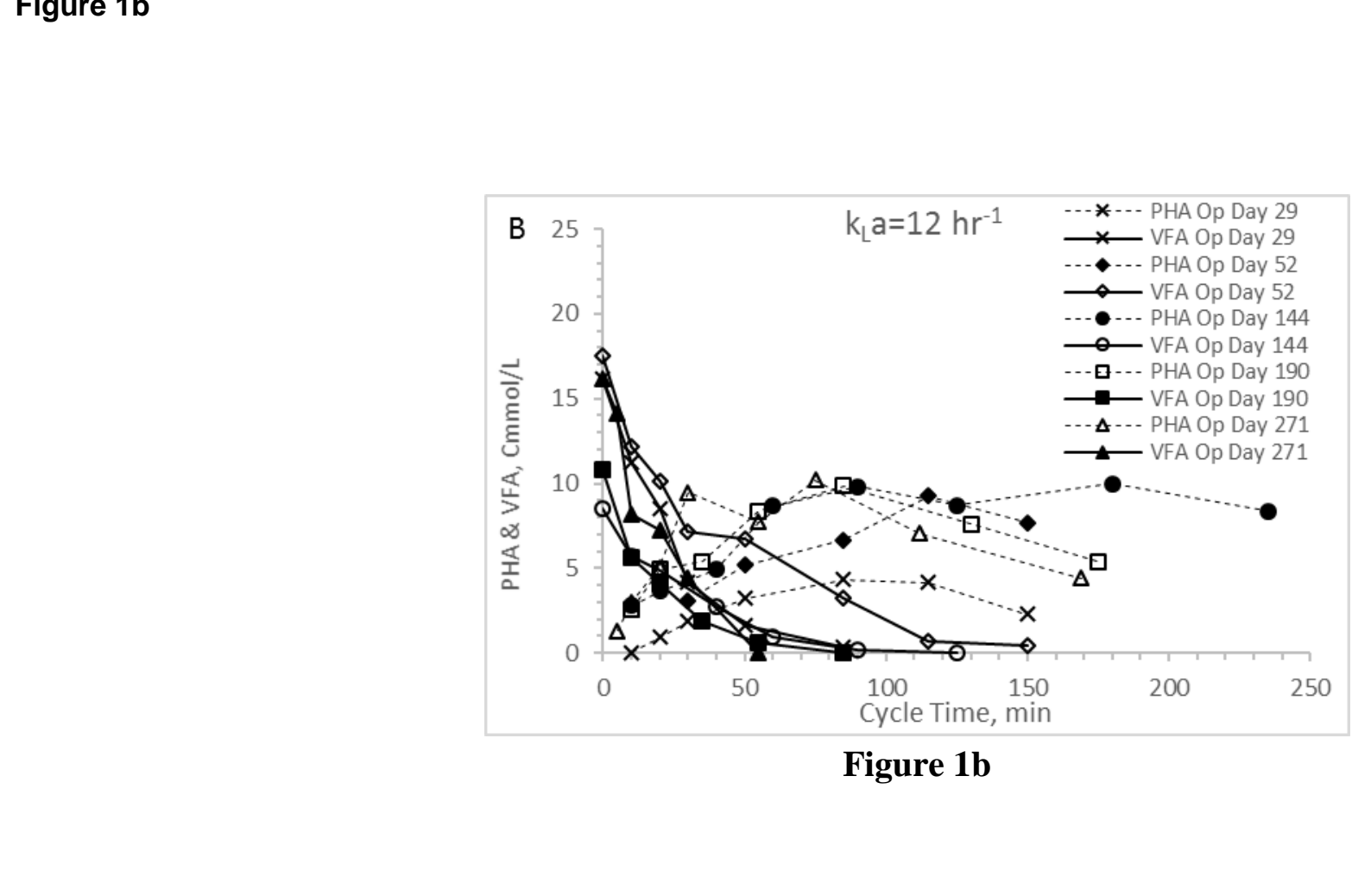

Figure 1b

(2)

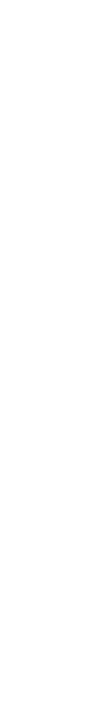

.
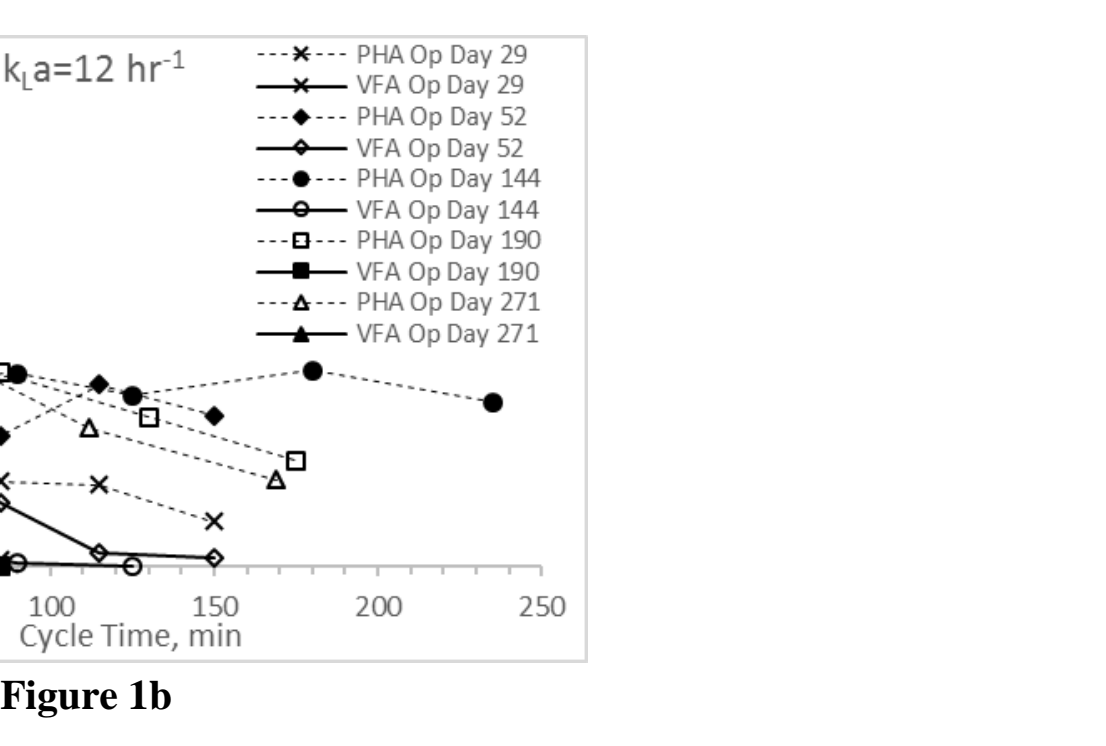

.

.

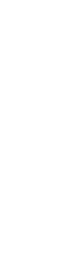

(n)
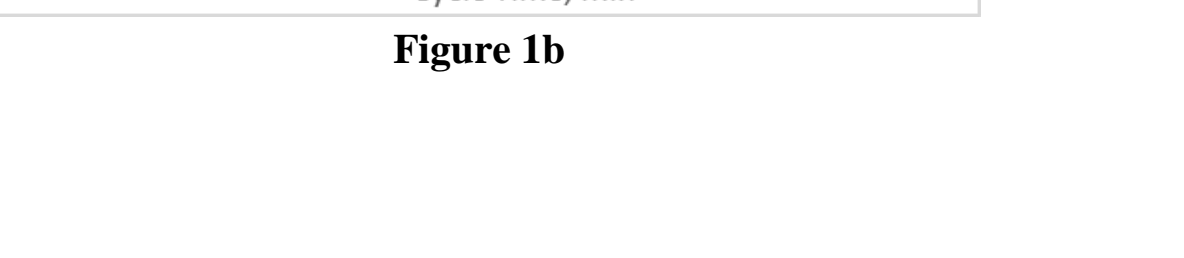
Figure 1c

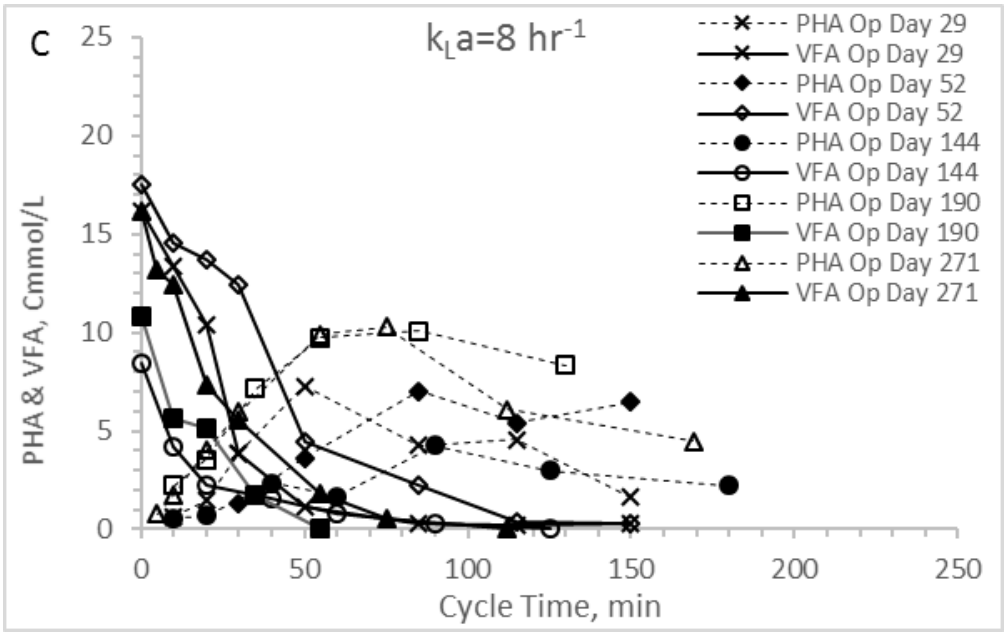

\section{Figure 1c}


Figure 1d

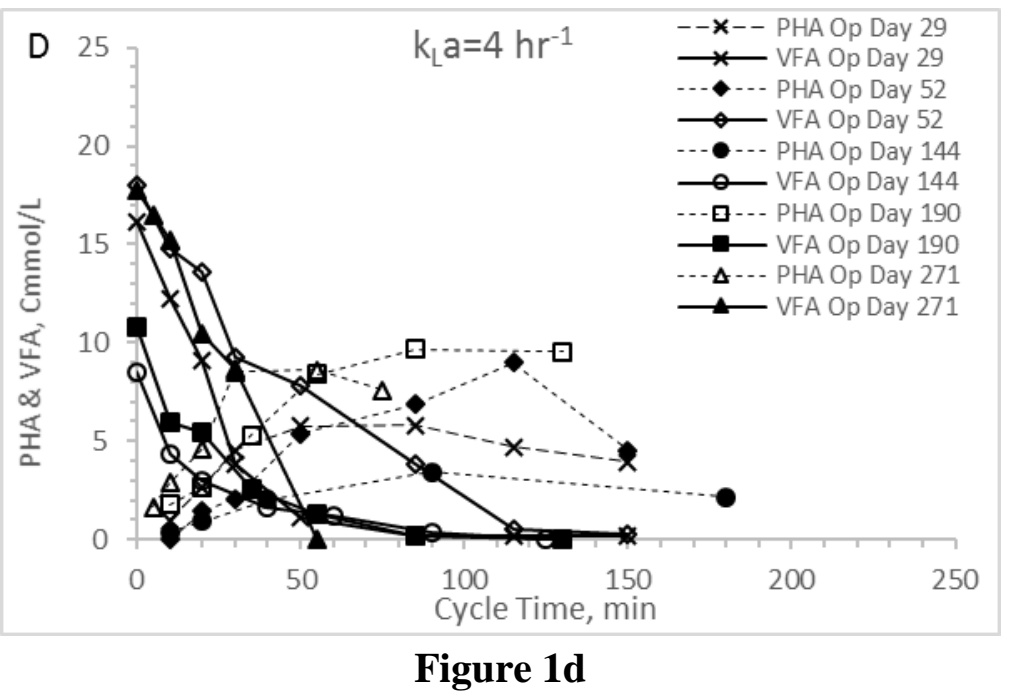

Figure 1d

Figure 1d

Figure 1d

Figure 1d

Figure 1d

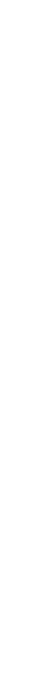


Figure $2 a$

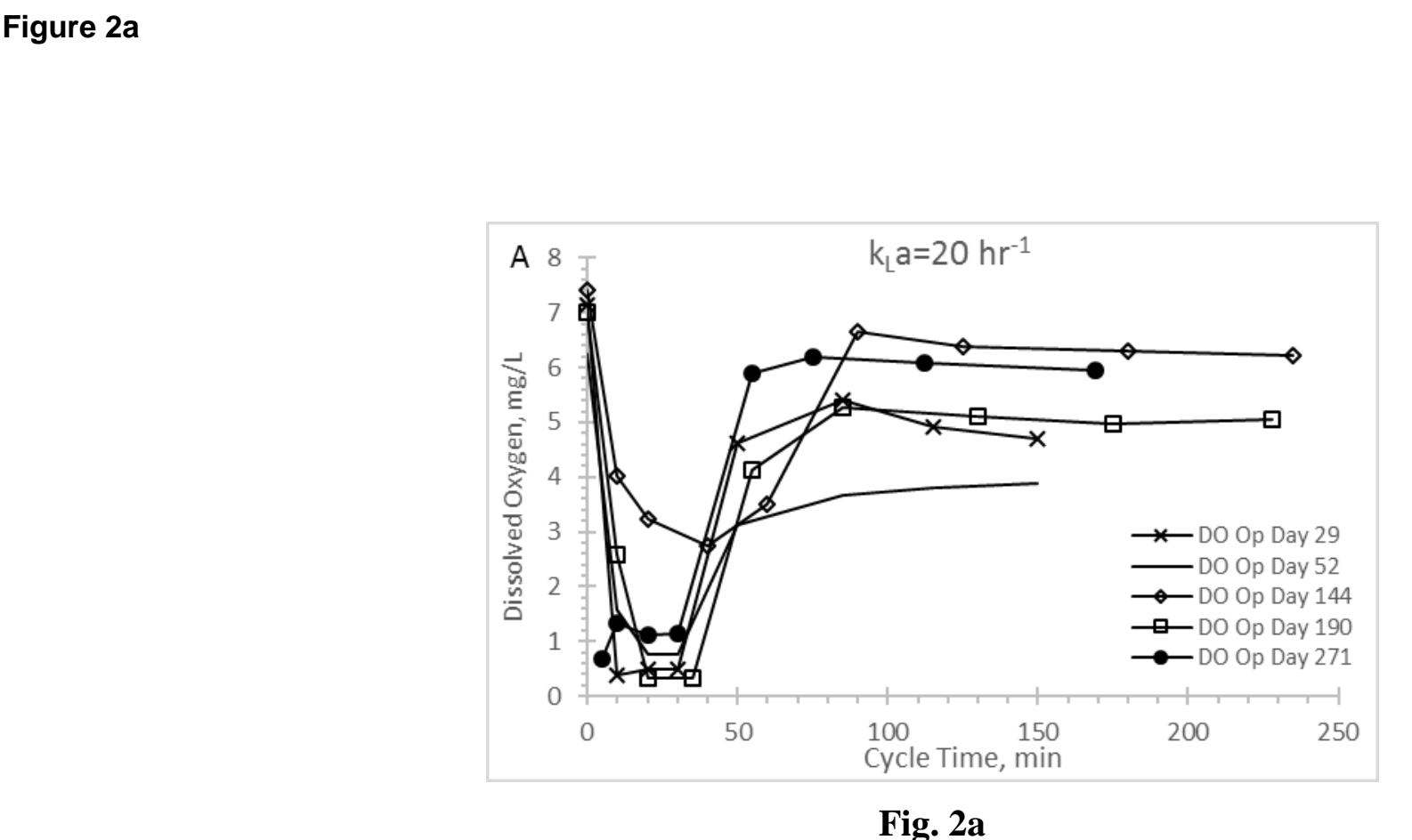

Fig. 2a

Figure $2 a$

政

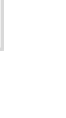
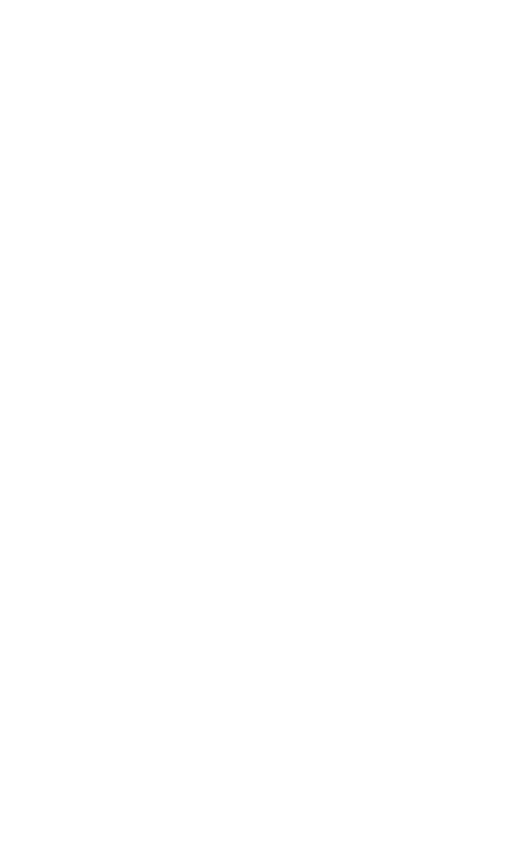
Figure 2b

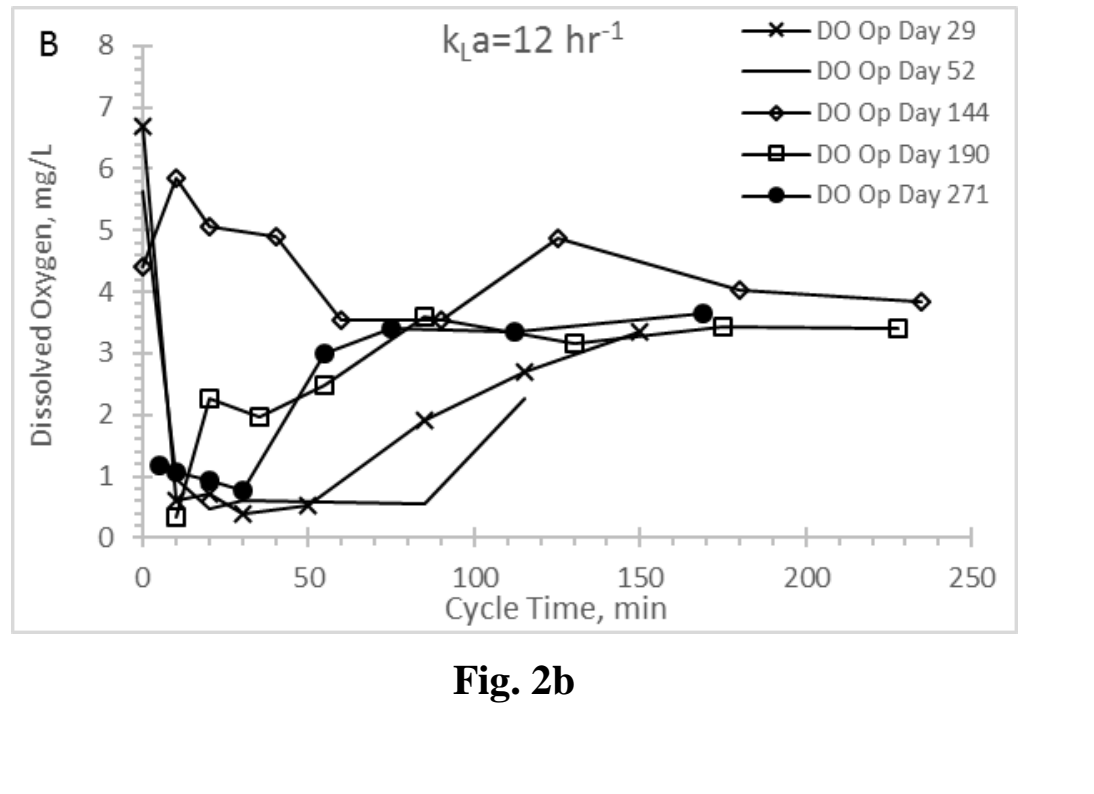

Fig. 2b

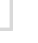

. $2 \mathrm{~b}$

(2)

(n)
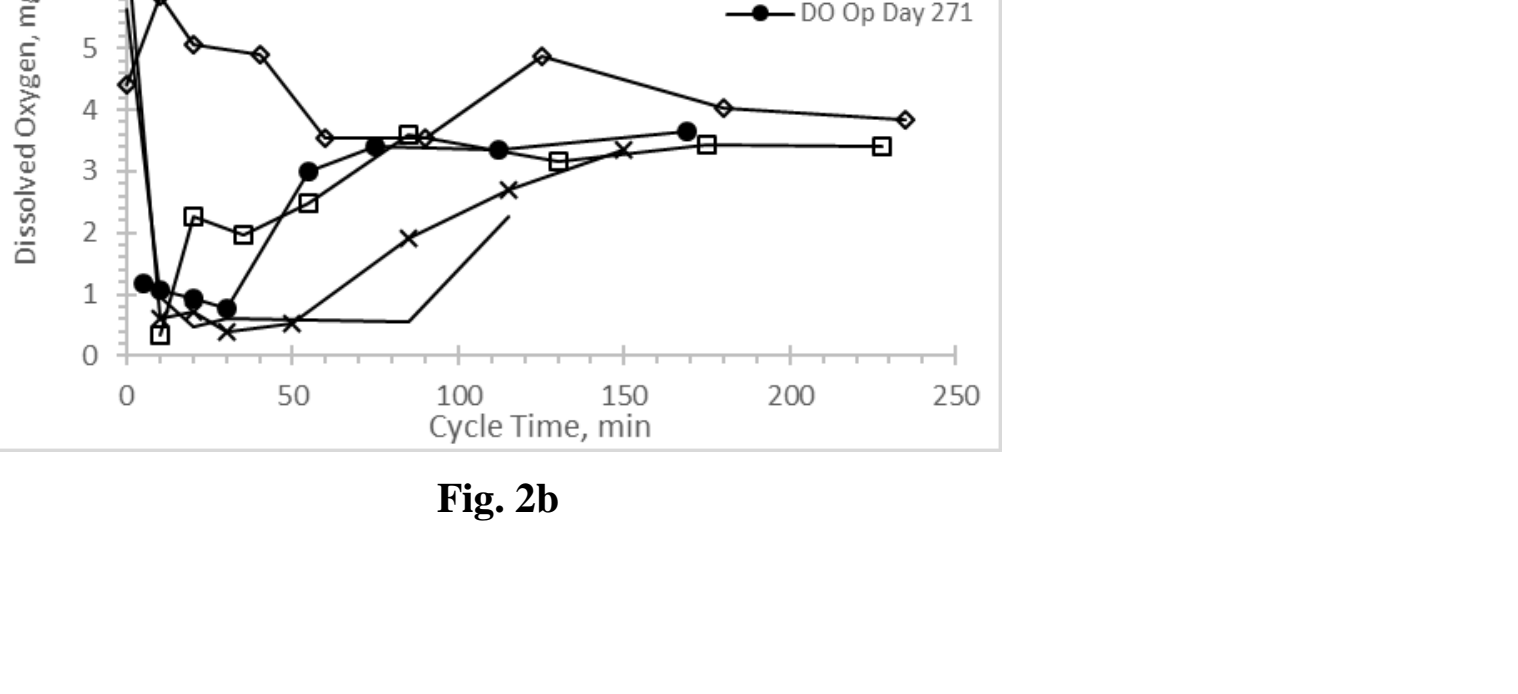

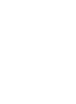

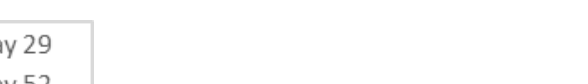

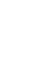

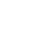

政

(




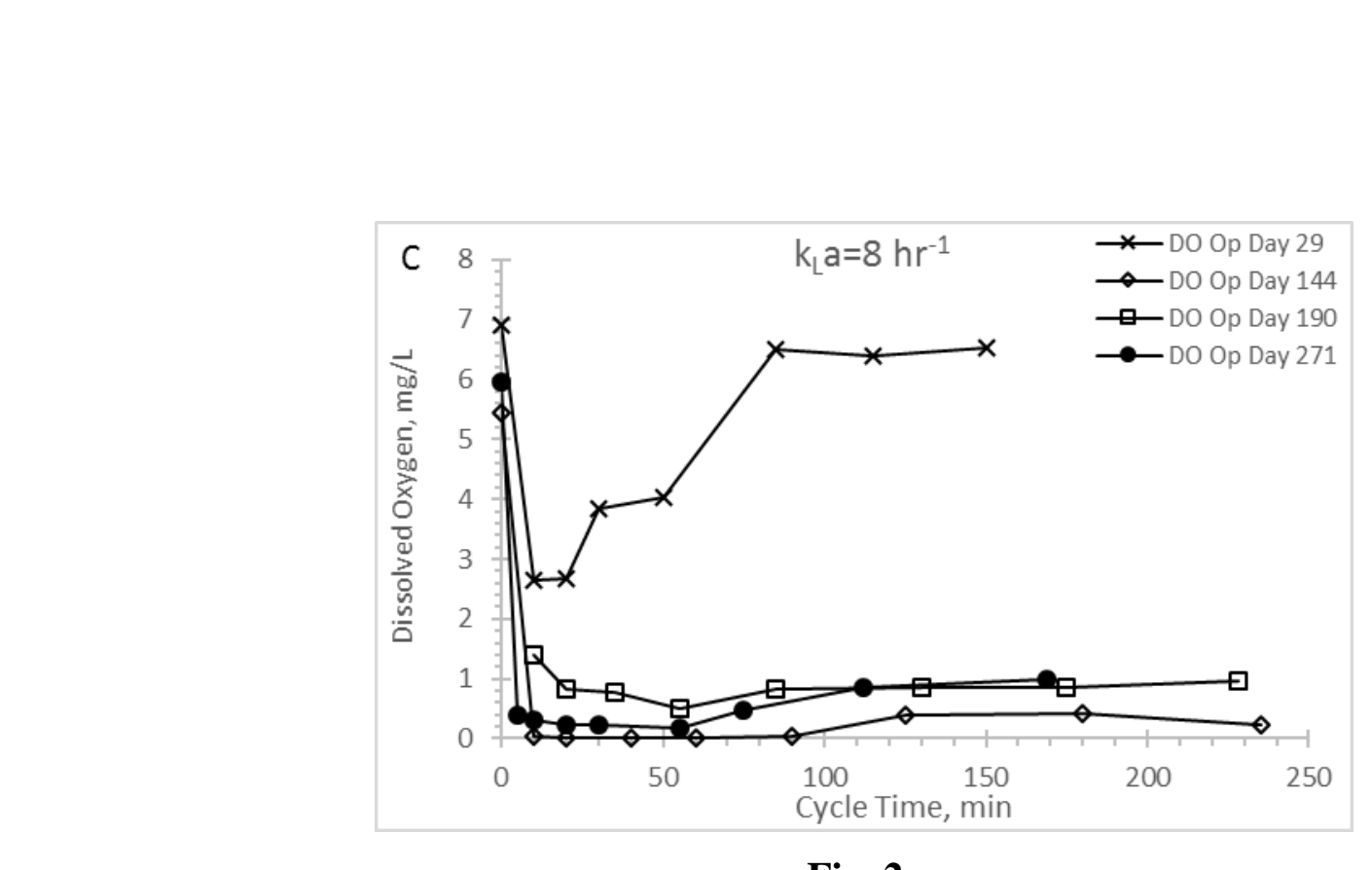

Fig. 2c

Figure 2c

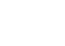

(a)

Figure $2 c$

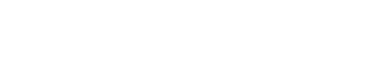
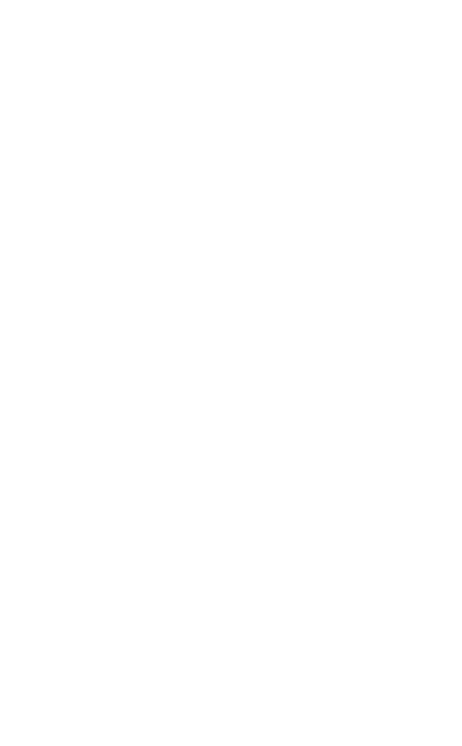

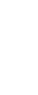

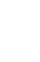
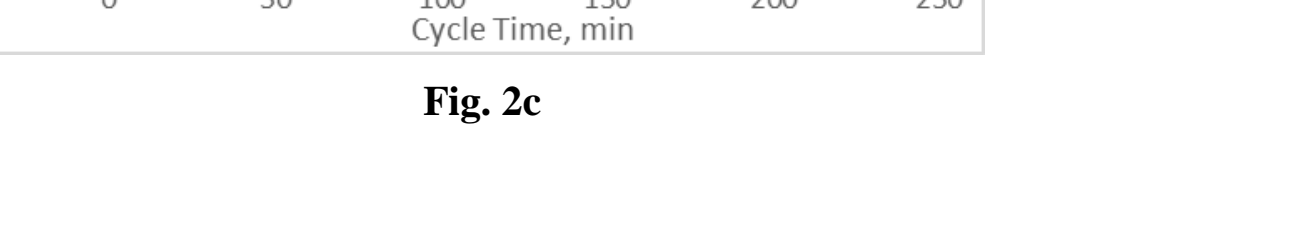
Figure 2d

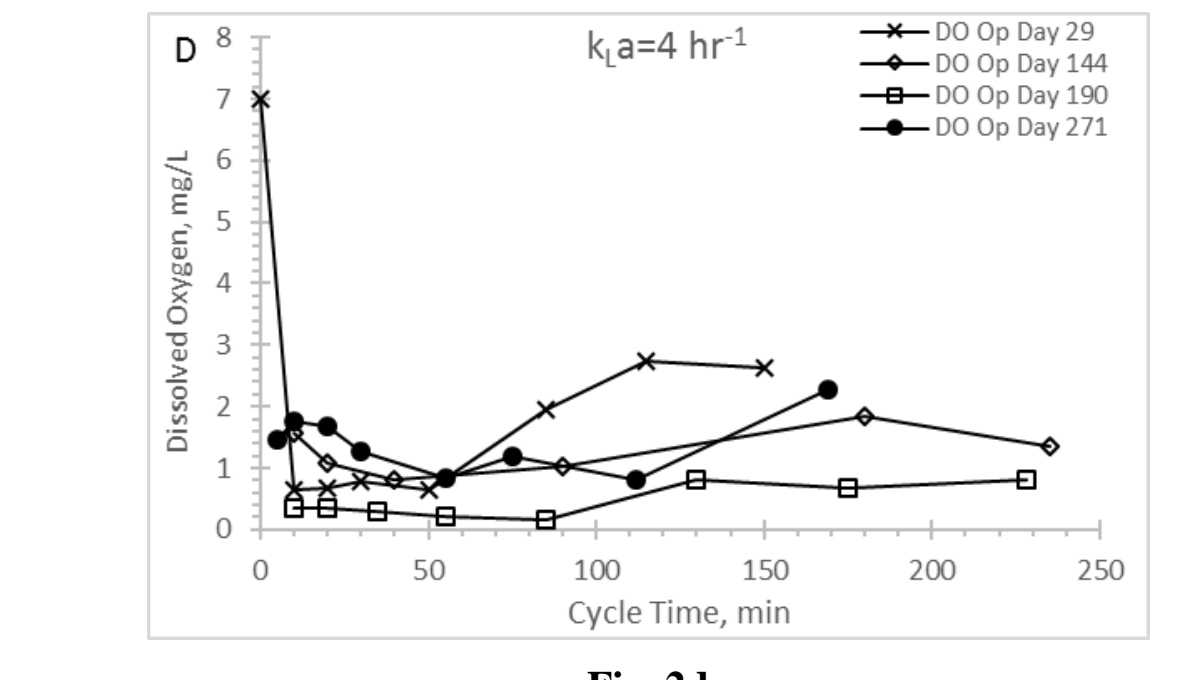

Fig. 2d

Figure 2d

(1)

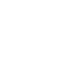

.

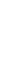


Figure 3a

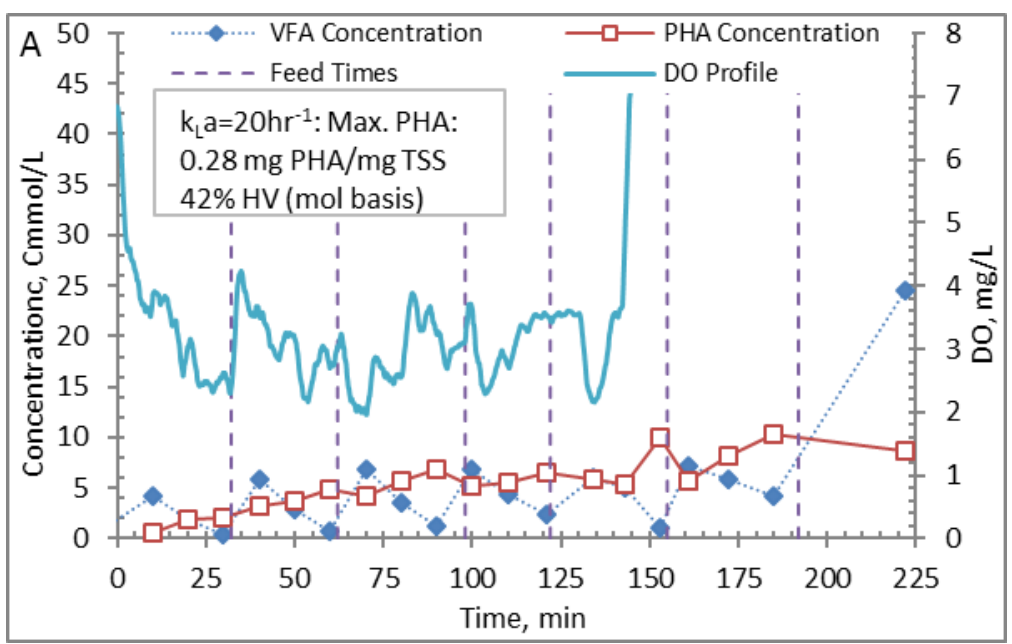

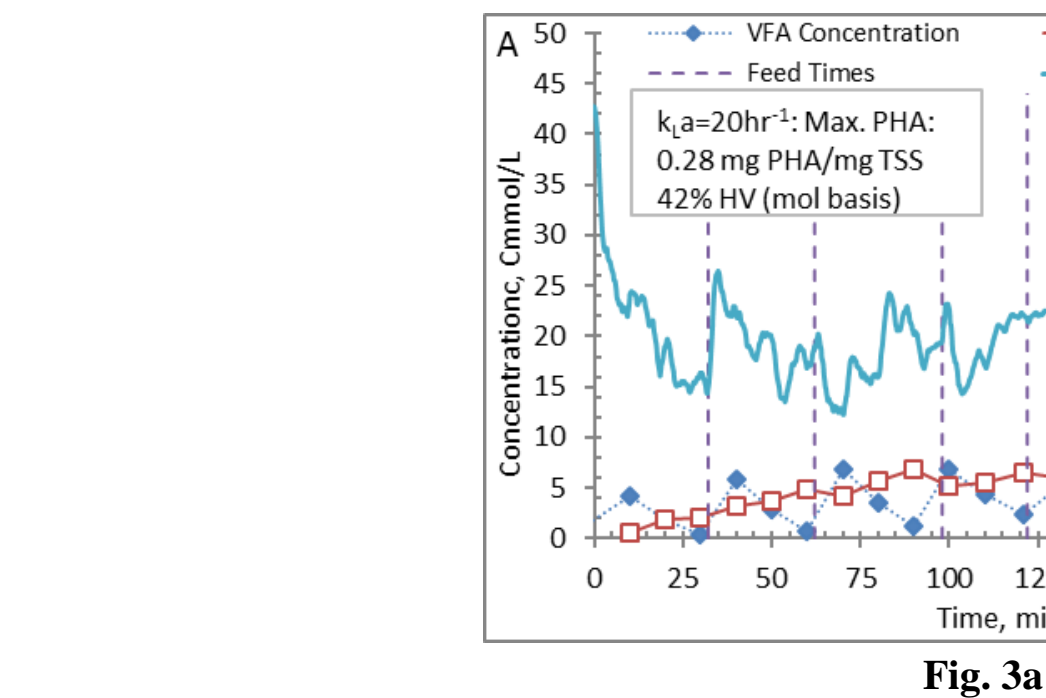

. $3 a$

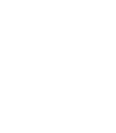

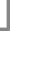

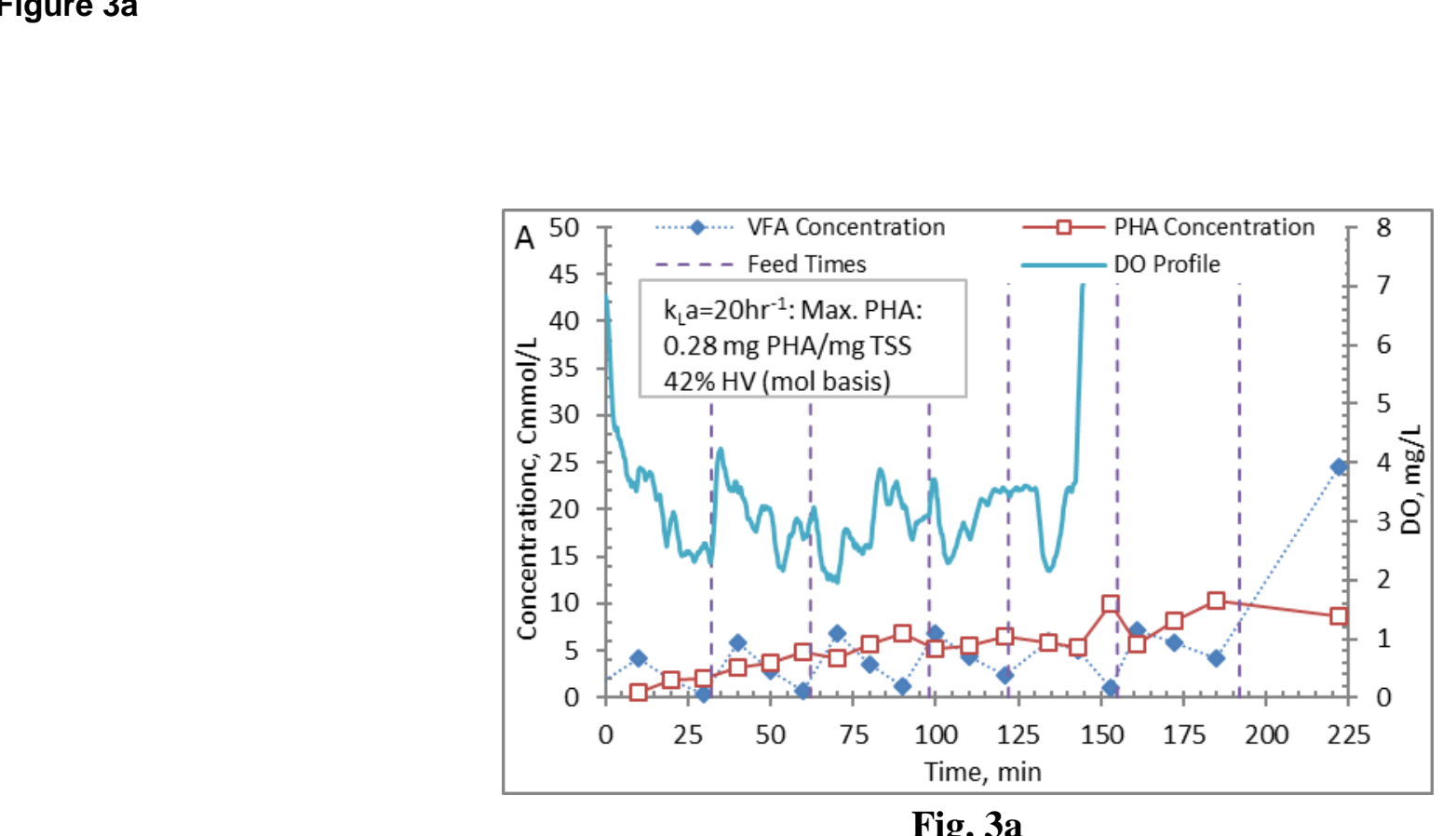
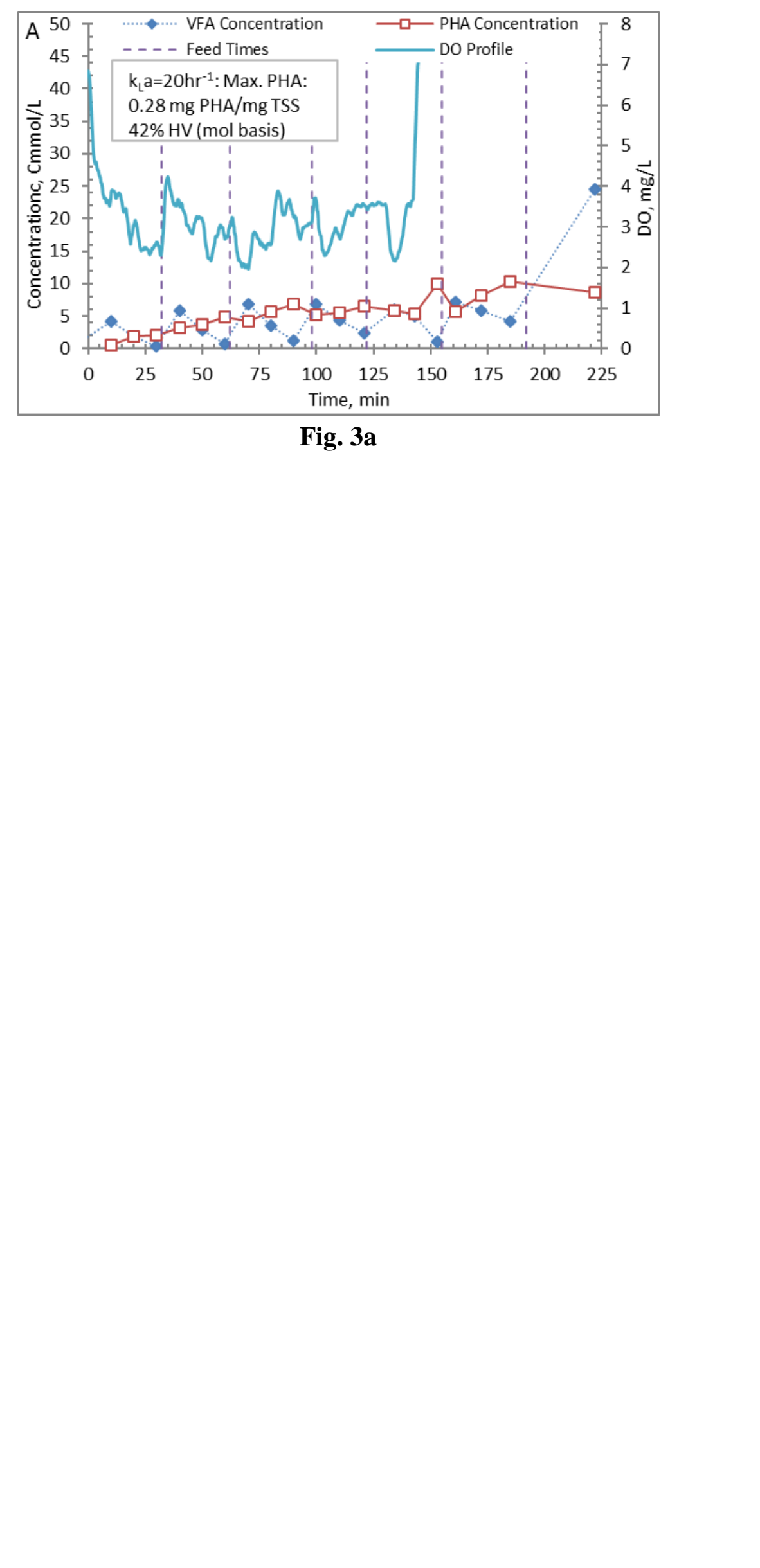
Figure $3 b$

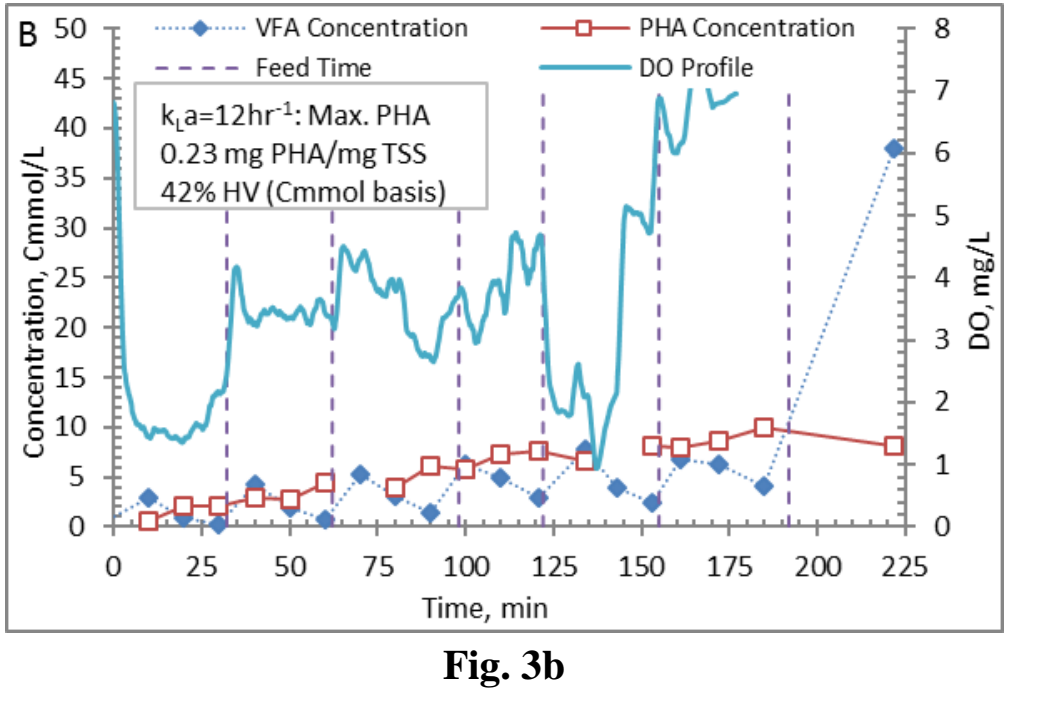

Fig. 3b

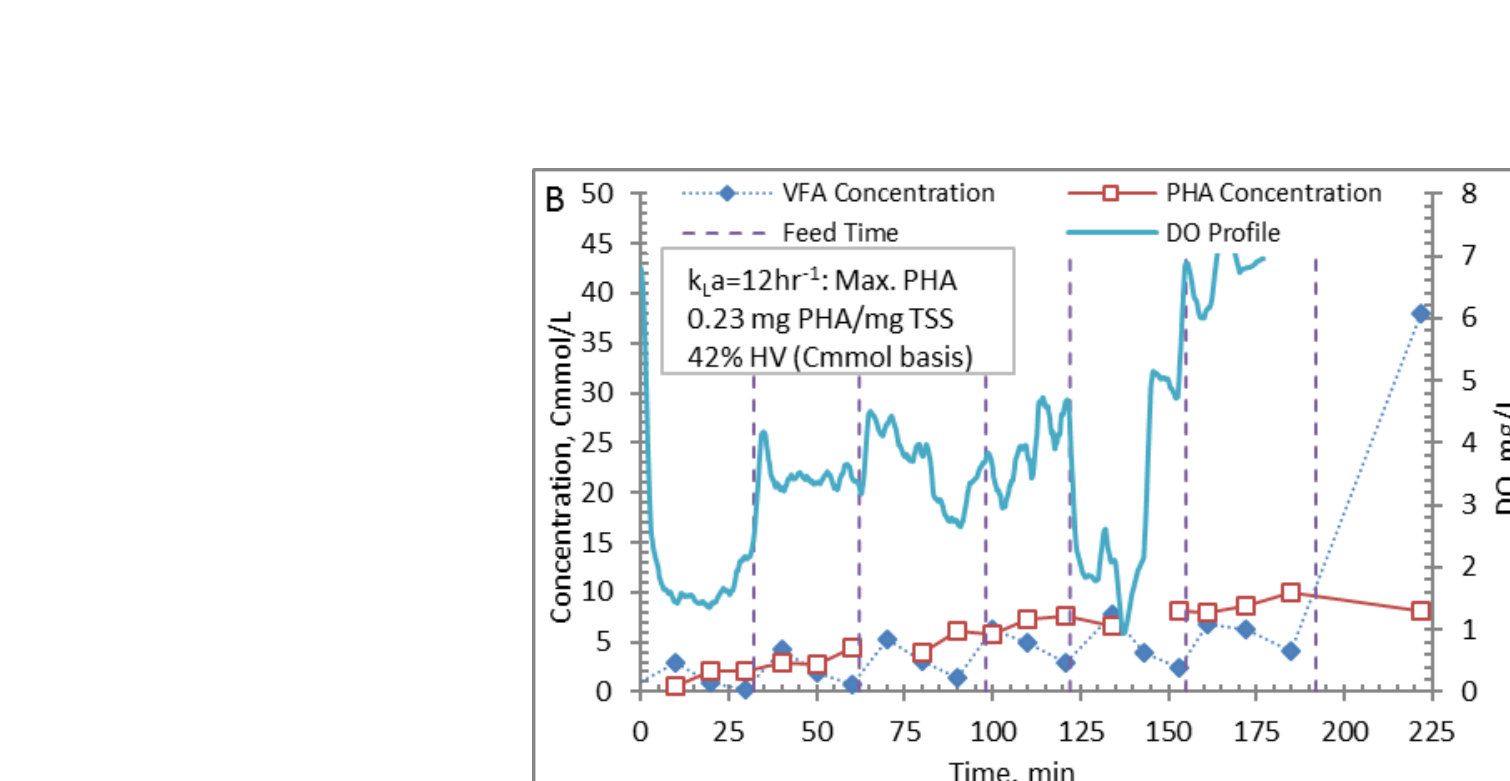

.

(n)
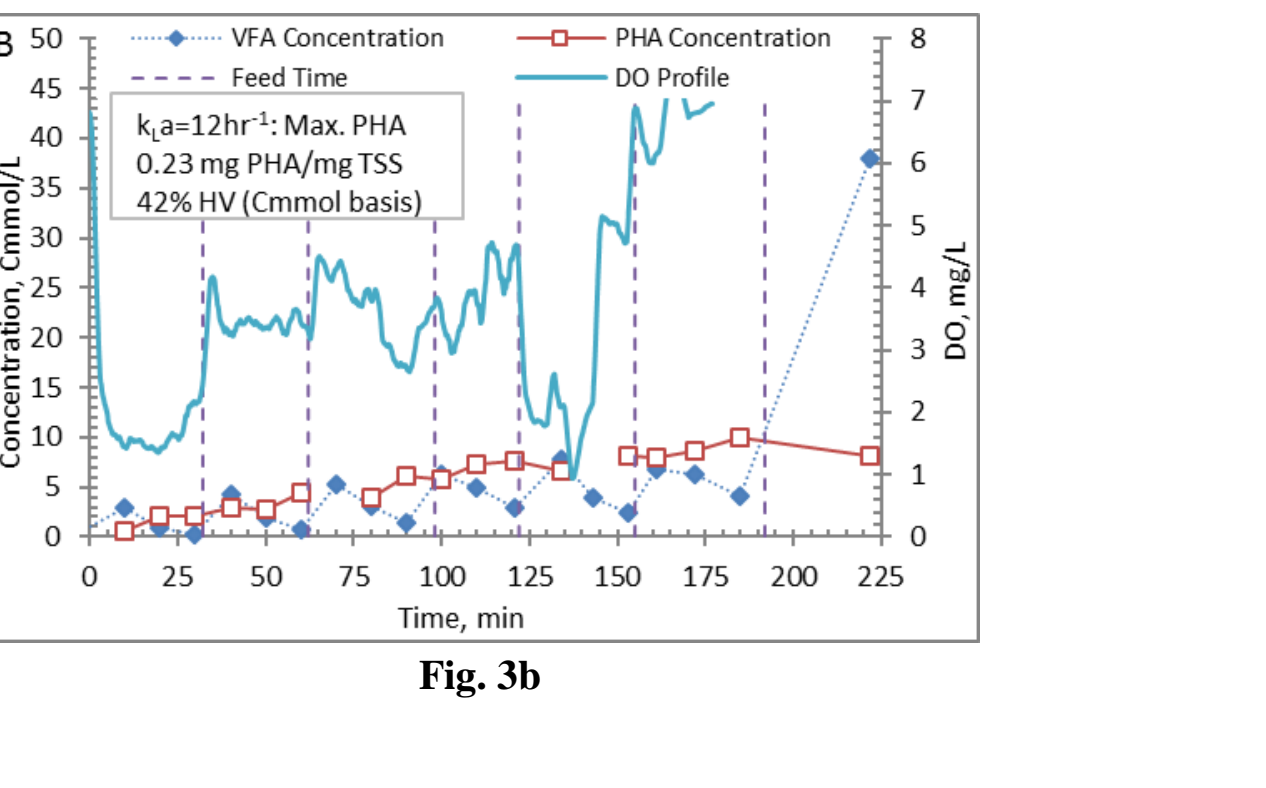
Figure 3c

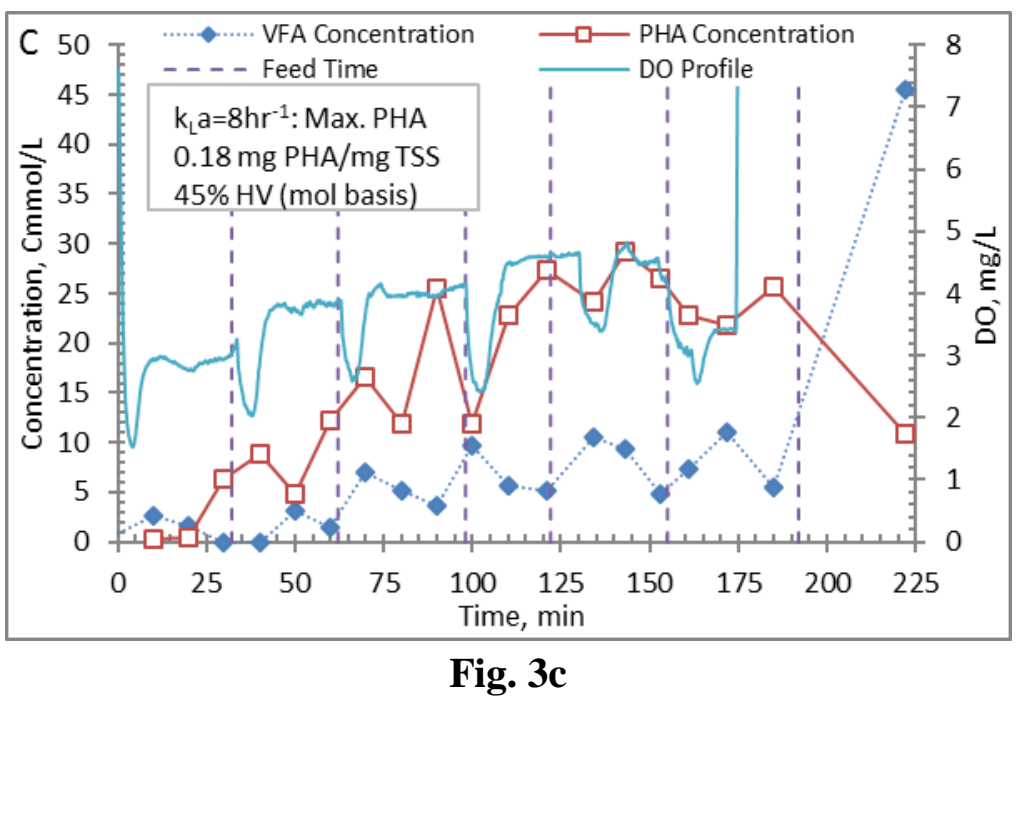

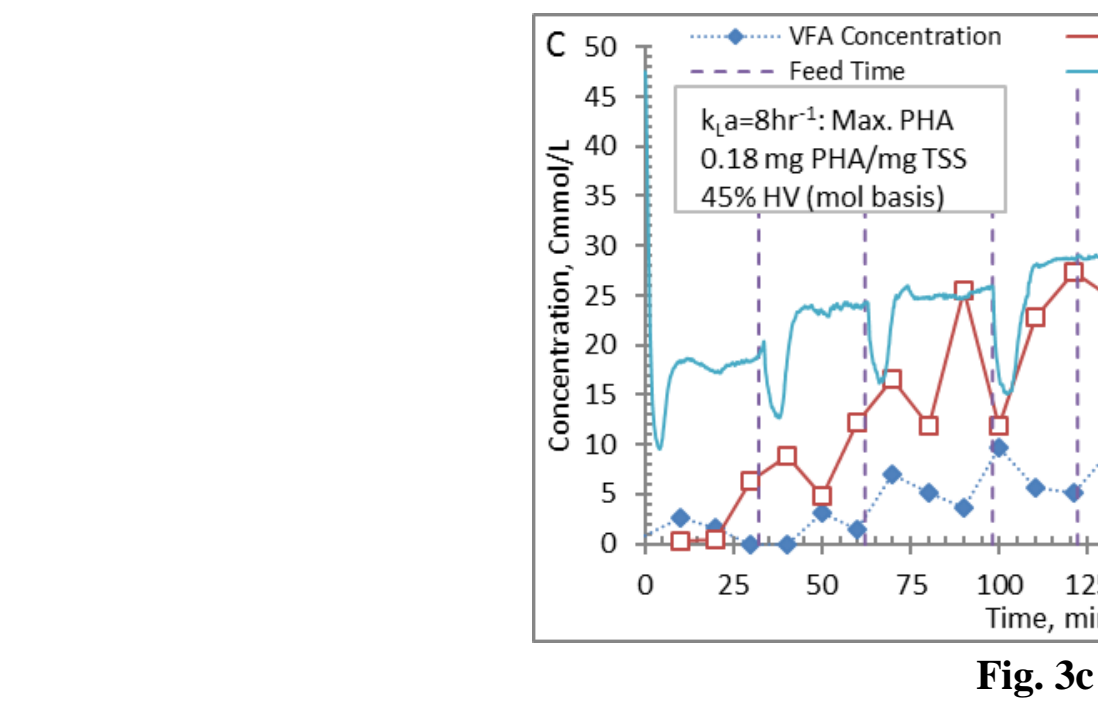

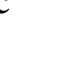

.

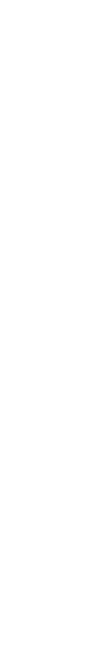

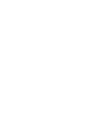

.
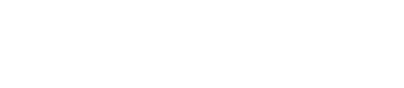
Figure 4a

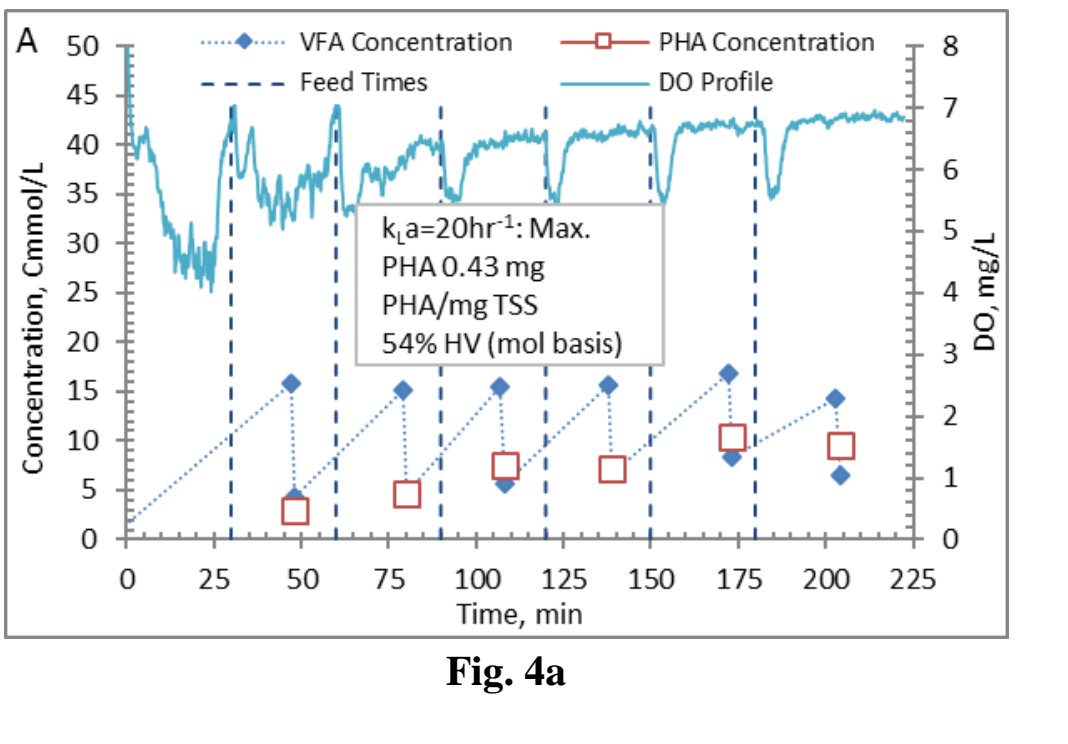

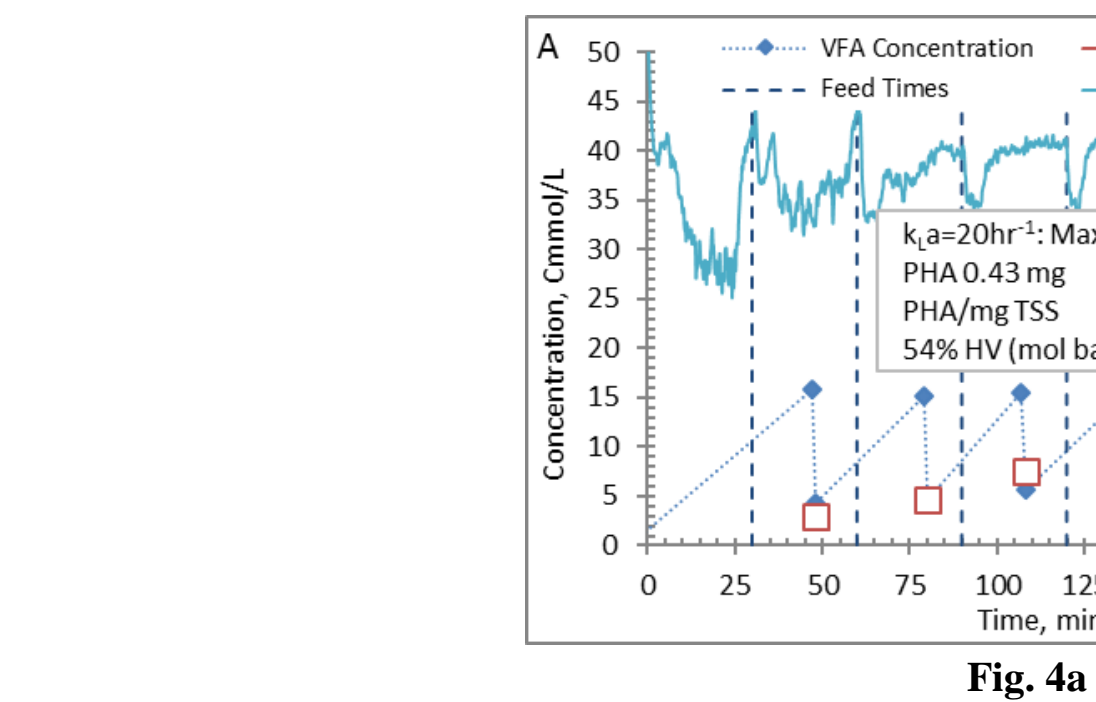

.

\section{Figure 4}

(1)

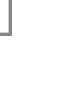
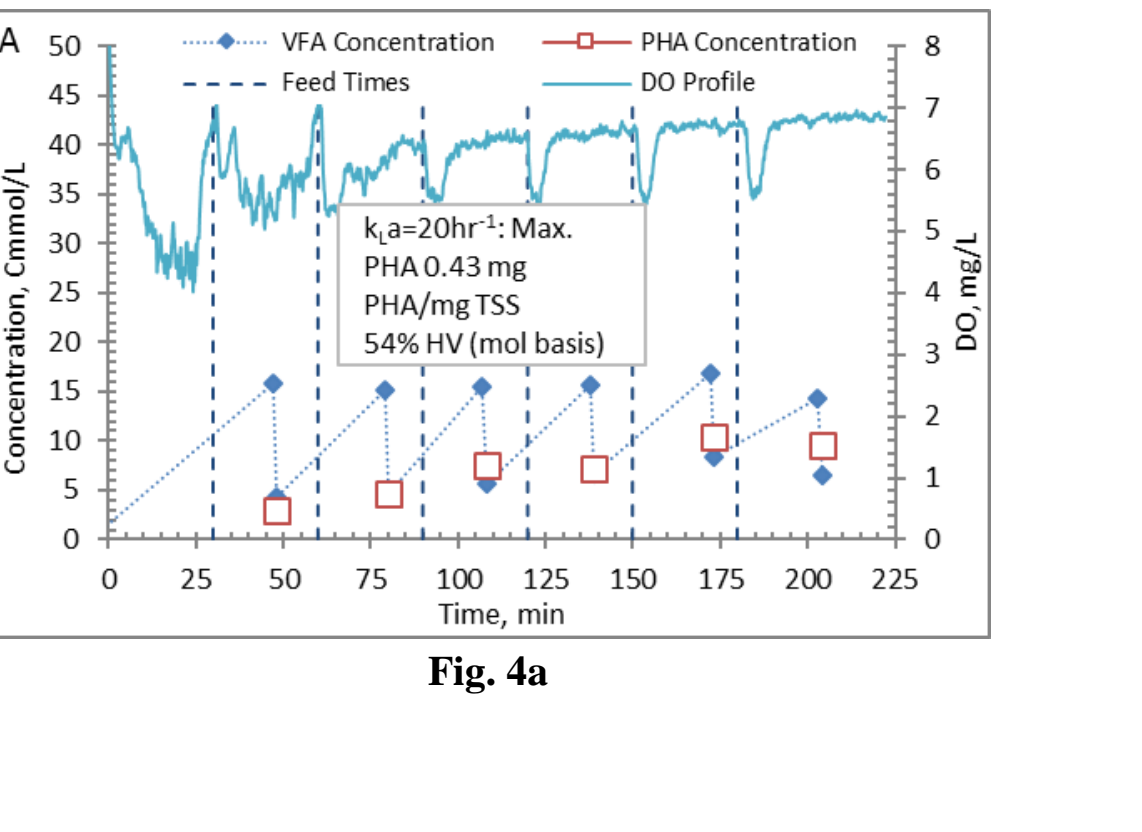

-

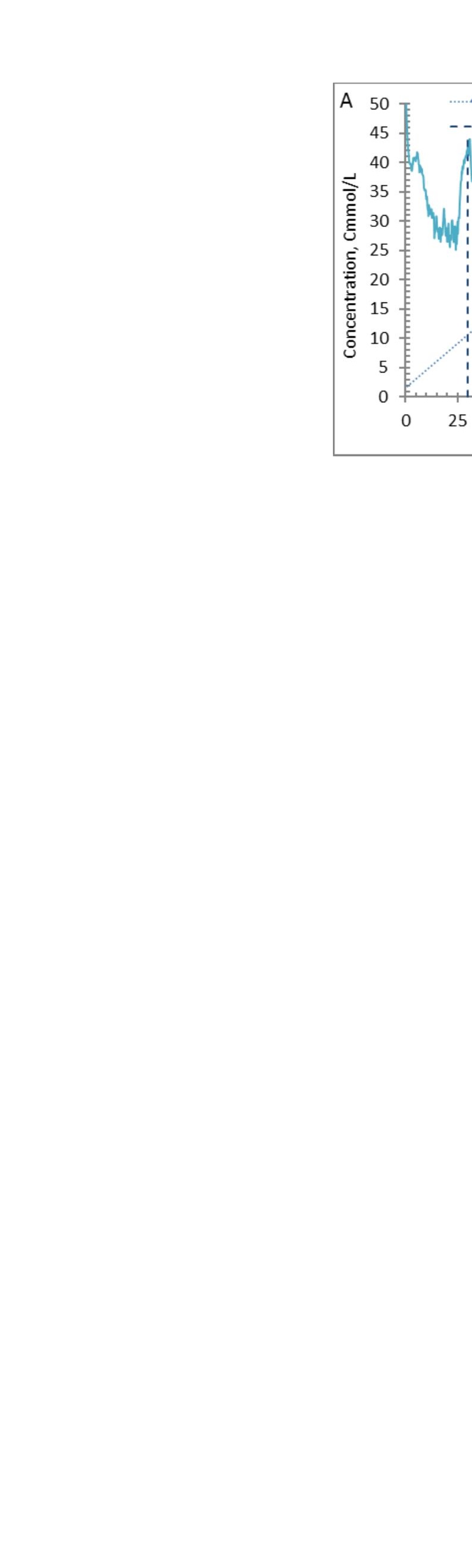

. 


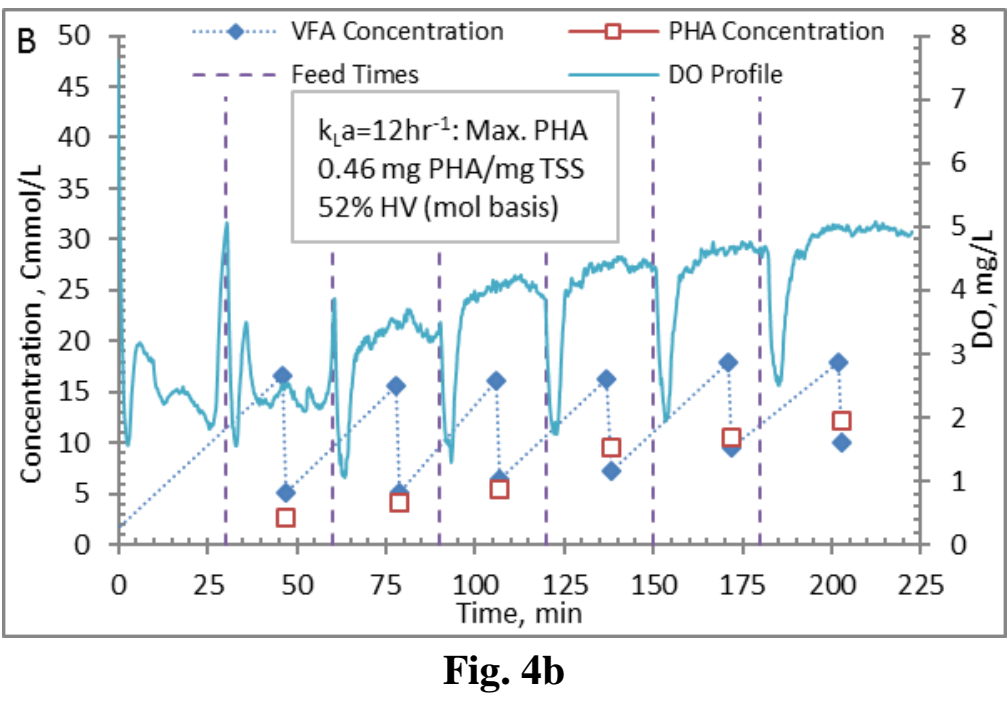

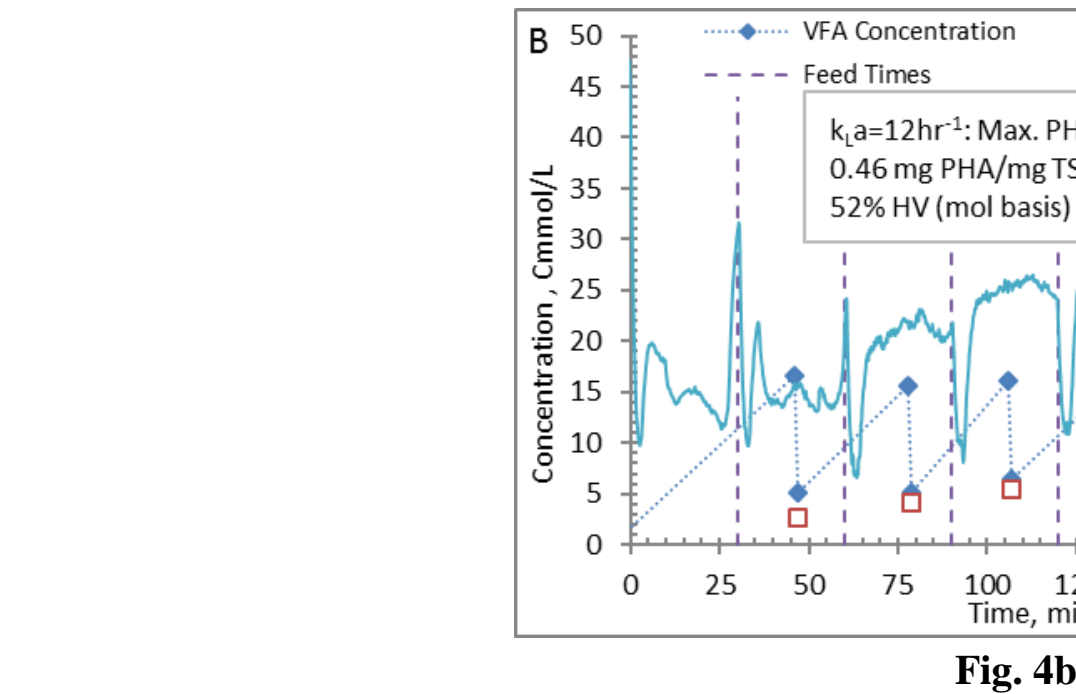

ig. $4 b$

.

fore 40

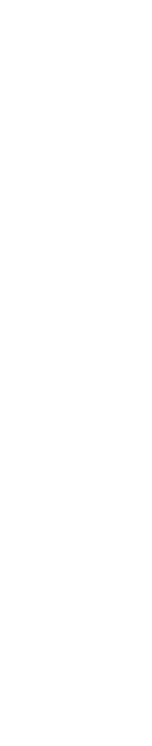
.

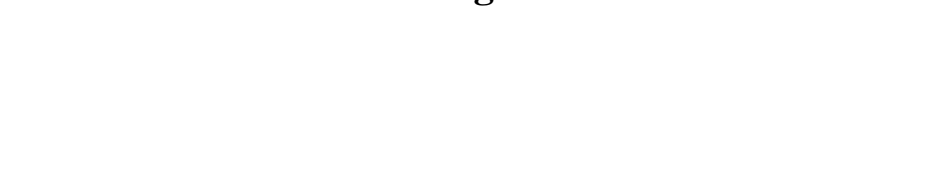


Figure $5 \mathrm{a}$

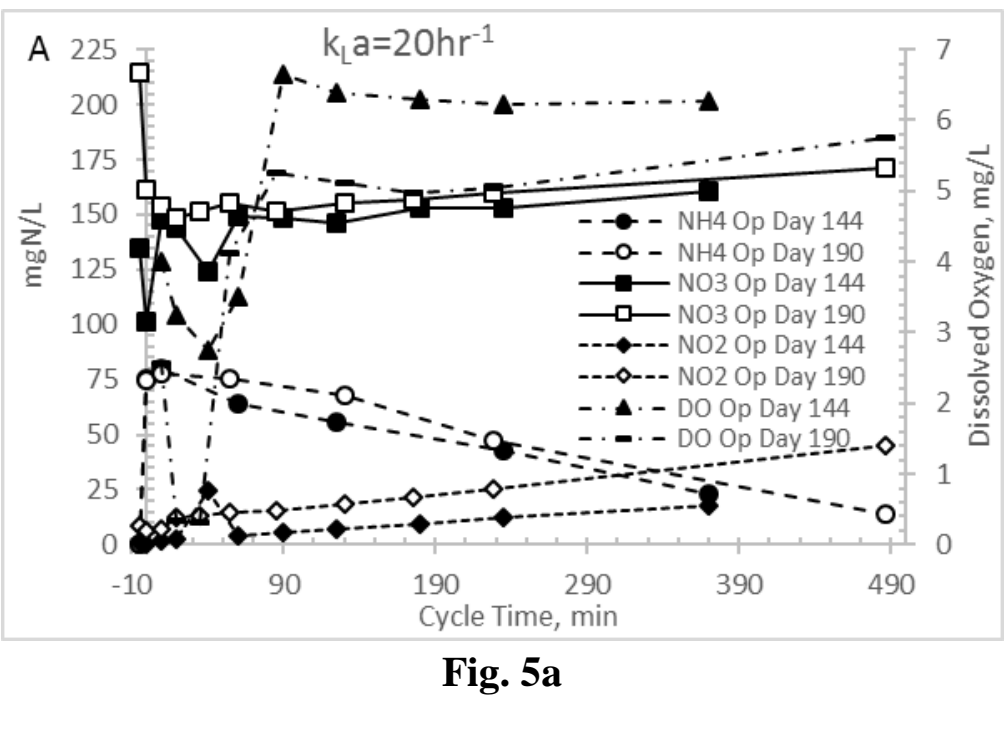

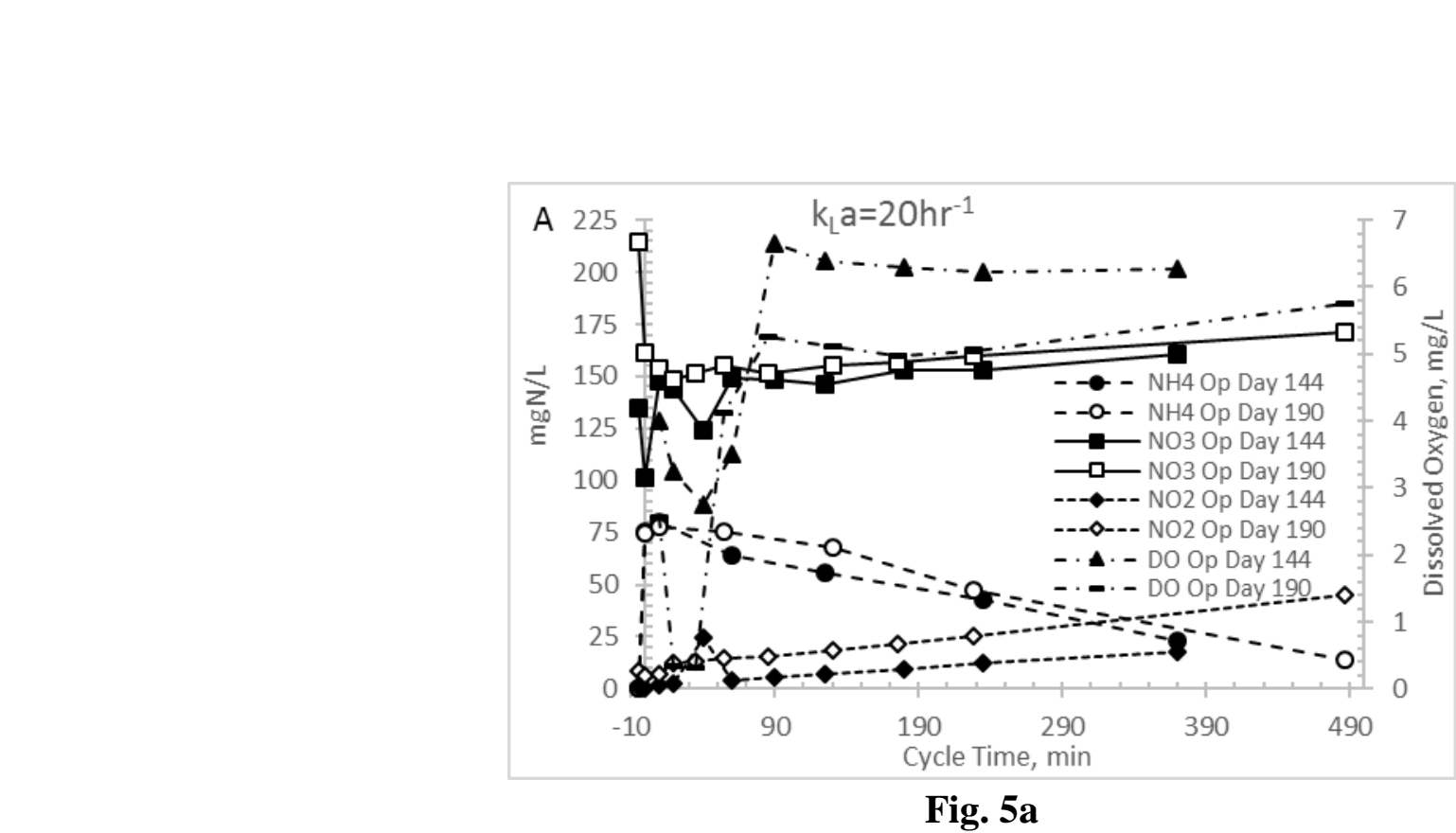

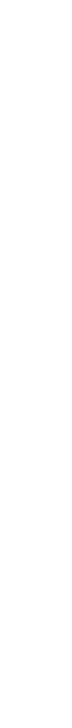

(n)

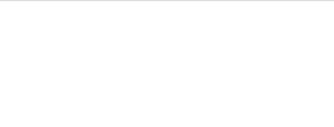
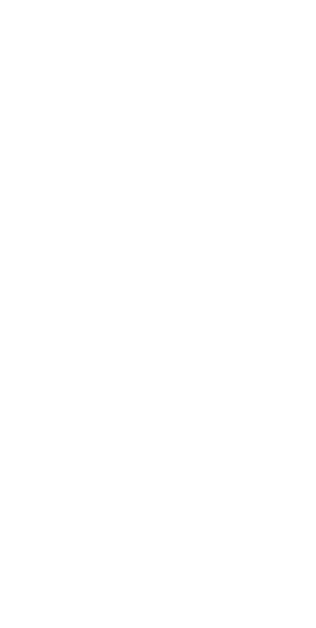

.

$$
\text { (n) }
$$

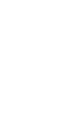

. .
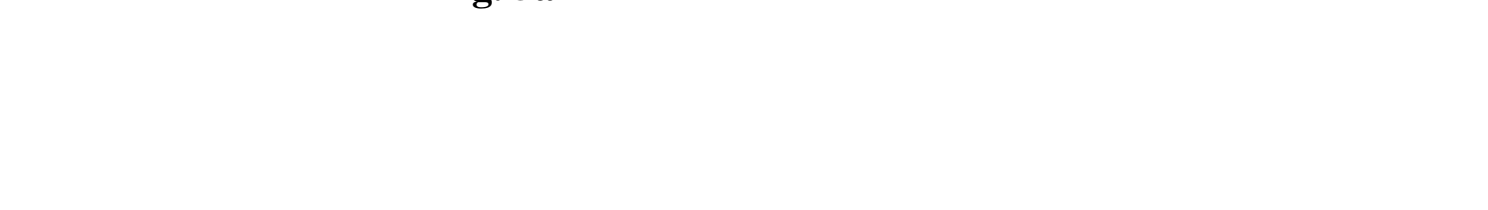

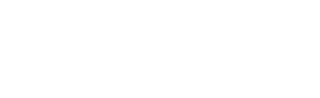
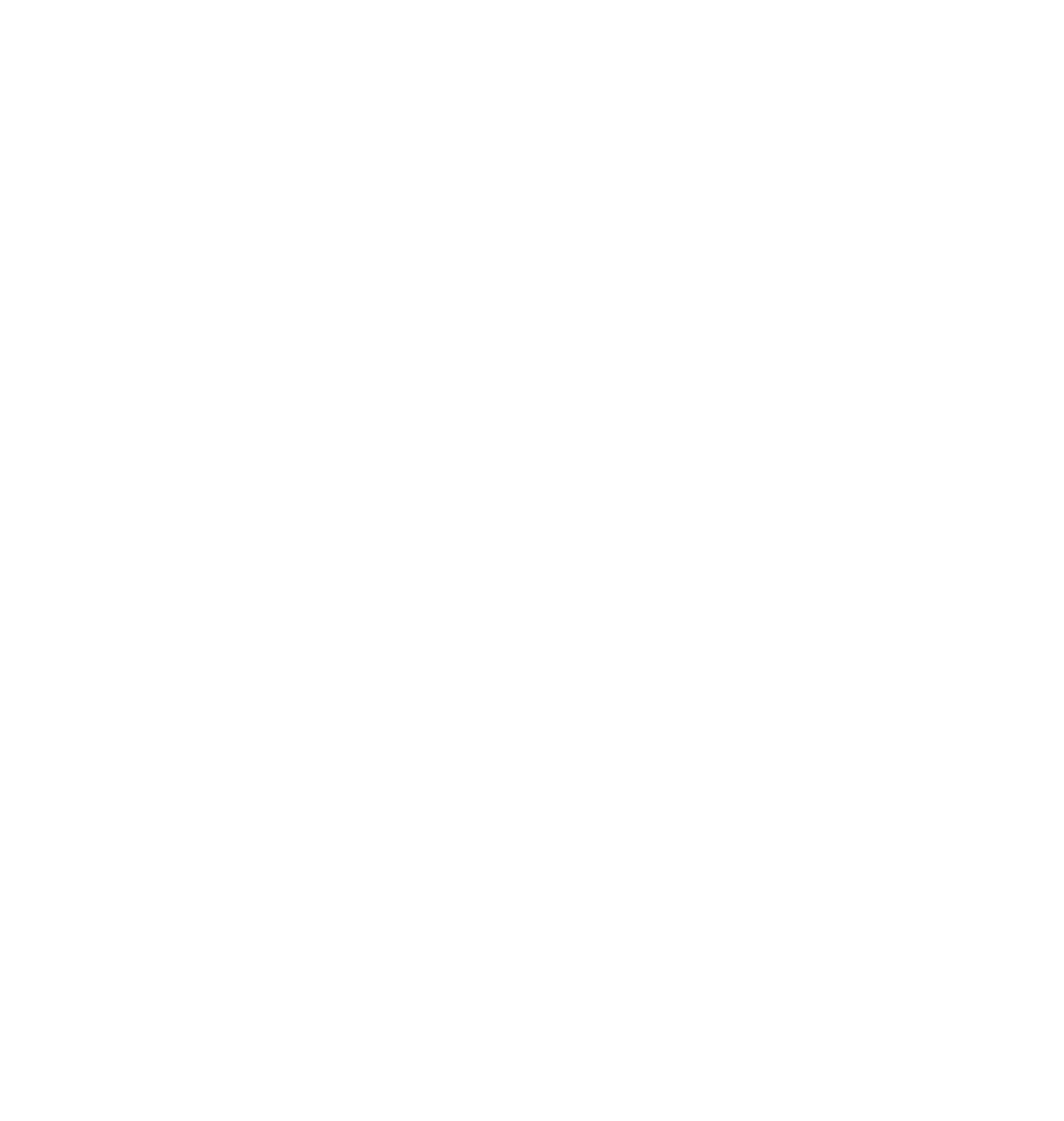


\section{B Figure 6b}

domain

Acidobacteria (1.1\%)

phylum

Actinobacteria (1.6\%)

Parcubacteria (1.0\%)

Firmicutes

$13.8 \%$

Planctomycetes (1.3\%)

Proteobacteria (48.8\%)

Spirochaetes (1.8\%)

Actinobacteria (1.6\%) Cytophagia (1.1\%) Flavobacteriia (1.6\%)

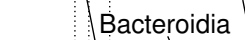

$\prod_{1}$

class

Bacteroidia Sp
Spirochaetia $(1.8 \%)$

Gammaproteobacteria (2.8\%)

Actinomycetales (1.1\%) Cytophagales (1.1\%) Rhodobacterales (1.4\%)

Bacteroidales / Flavobacteriales (1.6\%) Rhizọbiales |Burkholderiales $(2.1 \%)$ Spirochaetales (1.8\%)

order ||$|c|||$

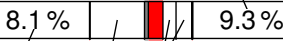

\rceil$>$ Rhodocyclales (25.6\%)

$9.64 \%$

$18.84 \%$ Sphingobacteriales $(2.1 \%)$ Clostridiales $/ 1.2 \%)$

Erysipelotrichales (4.3\%) Parcubacteria_genera_inc._sed. $(1.0 \%)$

\section{Prevotellaceae (1.3\%) Flavobacteriaceae (1.0\%)}

Porphyromonadaceae $(4.4 \%) /$ Saprospiraceae $(1.1 \%)$

Bacteroidaceae (2.1\%) Ruminococcaceae (3.3\%) Erysipelotrichaceae (4.3\%)

family

Parcubacteria_genera_inc._sed. $(1.0 \%)$

Planctomycetaceae $(1.2 \%)$

Methylobacteriaceae

Proteiniphilum (1.2\%) Parcubacteria_genera_inc._sed. (1.0\%)

Bacteroides (2.1\%)/Haliscomenobacter (1.1\%) - Meganema Thauera (1.2\%) Sphaerochaeta (1.5\%) 
Acidobacteria (1.1\%)

Firmicutes Planctomycetes (1.4\%)

Spirochaetes (1.2\%) Verrucomicrobia (1.9\%)

phylum \begin{tabular}{|l|l}
\hline Bacteroidetes (22.4\%) & $15.4 \%$ \\
\hline
\end{tabular} Proteobacteria (39.0\%)

Flavobacteriia (1.9\%) Sphingobacteriia (1.9\%) Erysipelotrichia (4.5\%)

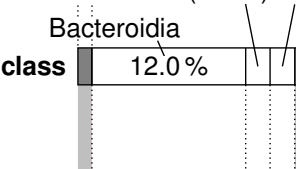

Clostridia Planctomycetia (1.3\%) Alphaproteobacteria

Flavobacteriales (1.9\%) Sphingobacteriales (1.9\%) Erysipelotrichales $(4.5 \%)$

\begin{tabular}{|c|c|}
\hline & $120 \%$ \\
\hline
\end{tabular}
Clostridiales

\begin{tabular}{l|l|l|l|l|l|}
\hline $9.4 \%$ & 7 & $13.5 \%$ & $18.4 \%$
\end{tabular}

\section{Betaproteobacteria} 1

Deltaproteobacteria (1.7\%) Gammaproteobacteria (4.3\%)

Porphyromonadaceae Prevotellaceae $(1.8 \%)$ Bacteroidaceae $(1.5 \%)$ Flavobacteriaceae $(1.5 \%)$

family

$6.4 \%$

Ruminococcaceae $(4.1 \%)$ Erysipelotrichaceae (4.5\%) Planctomycetaceae $(1.3 \%)$ 
phylum Bacteroidetes (25.4\%)

$16.7 \%$

Proteobacteria (34.9\%)

Flavobacteriia (2.5\%) Sphingobacteriia (1.5\%) Alphaproteobacteria Deltaproteobacteria (3.2\%)

$7.8 \%$

$21.0 \%$

1

Spirochaetia $(2.6 \%)$

Flavobacteriales (2.5\%) Sphingobacteriales (1.5\%) Rhizobiales Burkholderiales $(2.0 \%)$

Bacteroidales / Clostridiales Erysipelotrichales//Rhodocyclales Spirochaetales (2.6\%) order $14.2 \%$ $8.4 \% \quad 6.4 \% \mid 5.8 \%$ $18.1 \%$

Porphyromonadaceae Prevotellaceae (1.1\%)
Bacteroidaceae $(2.4 \%)$ Flavobacteriaceae $(1.8 \%$
inococcaceae $(3.4 \%)$

family

\begin{tabular}{|l|l|l|}
\hline & $6.4 \%$ \\
\hline pelotrichaceae
\end{tabular}

Ru $18.1 \%$

Methylobacteriaceae (5.0\%)

Rhodocyclaceae Spirochaetaceae $(2.2 \%$

Thauera Zoogloea Sphaerochaeta (2.0\%)

Bacteroides (2.4\%) Proteiniphilum (3.0\%) Meganema (5.0\%) 


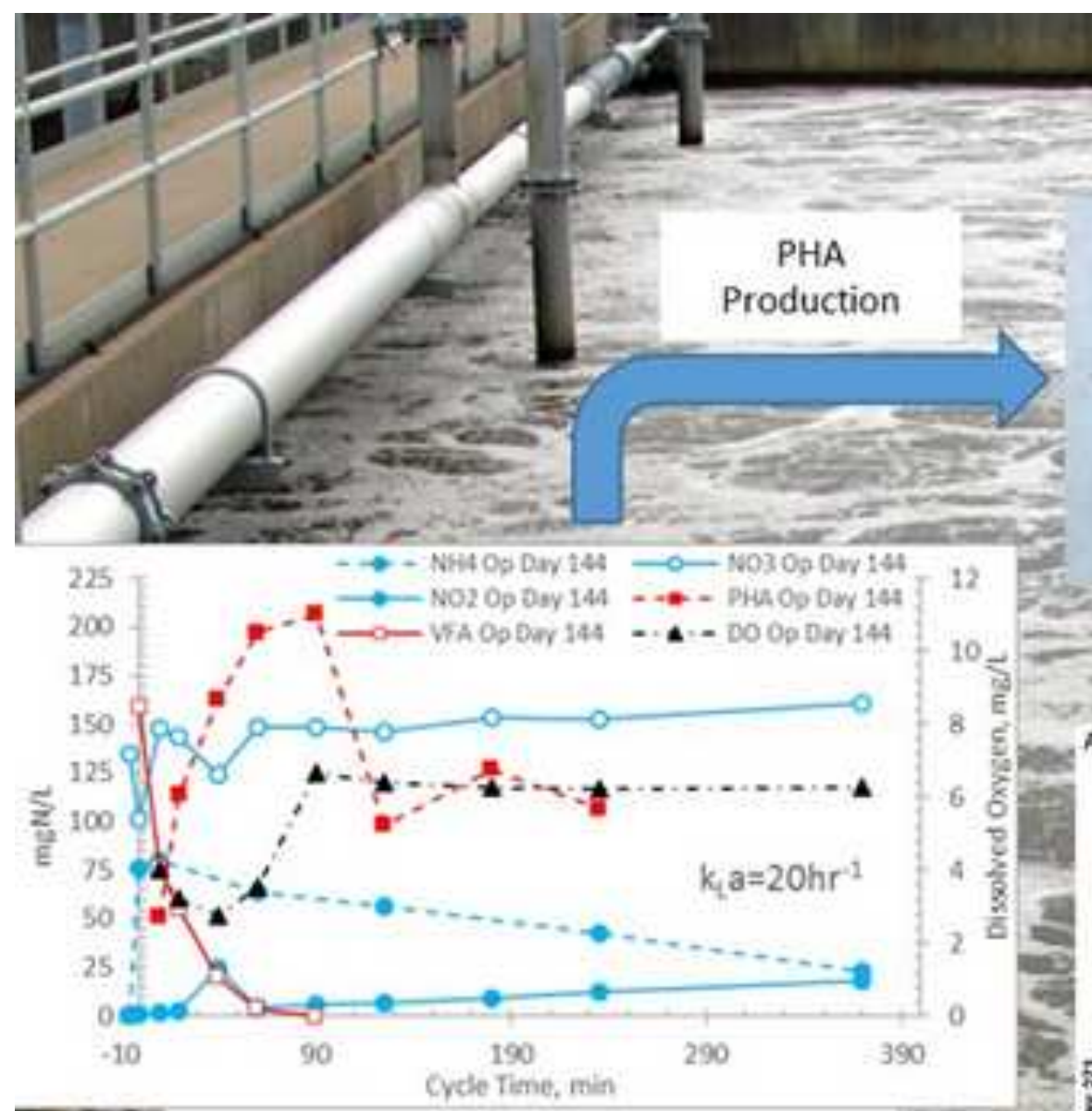

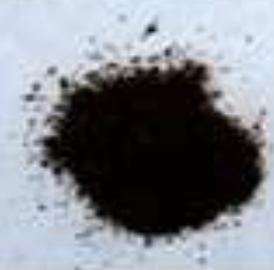

PHA-rich Biomass
Purified PHA

Extracted PHA

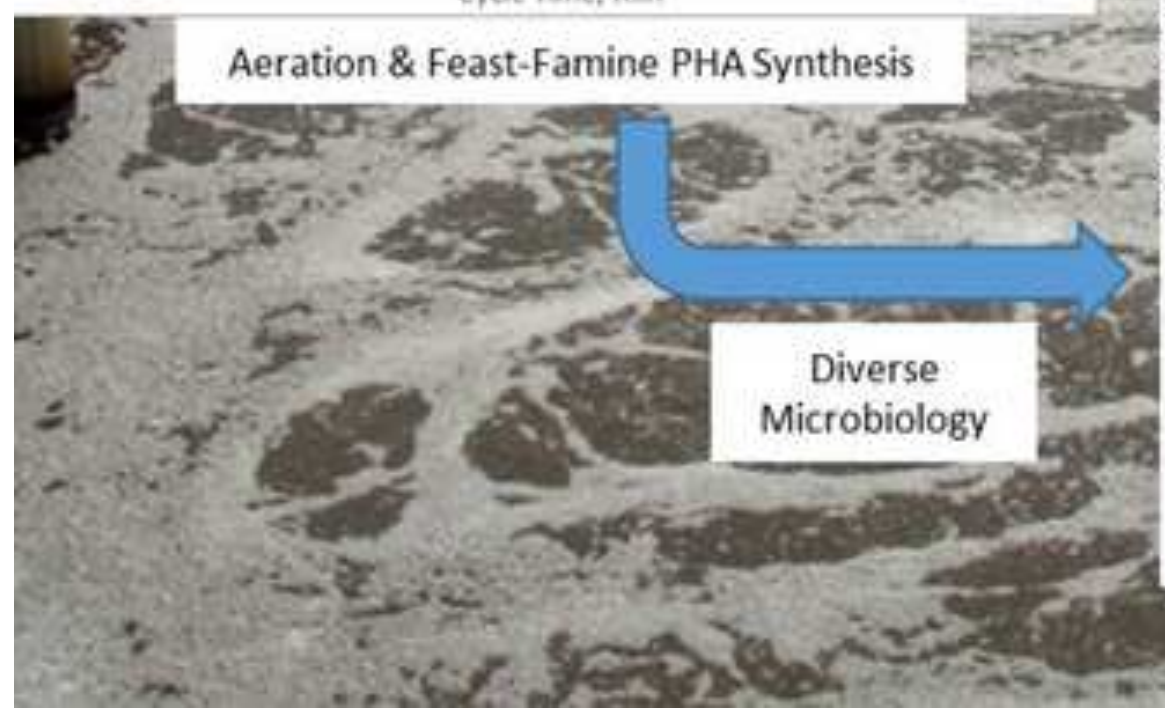

Q

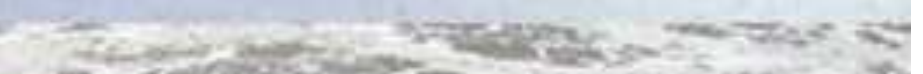
-

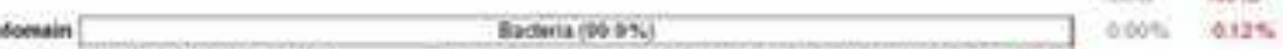

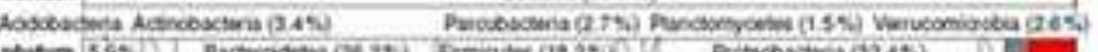

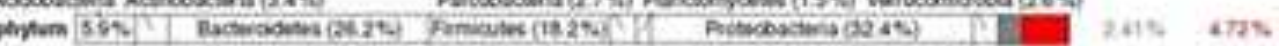

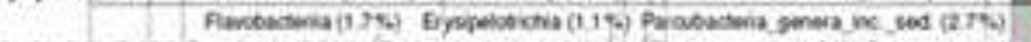

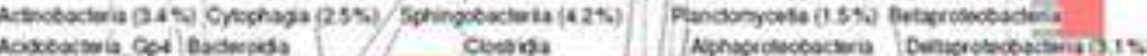
dessisty tios HTC

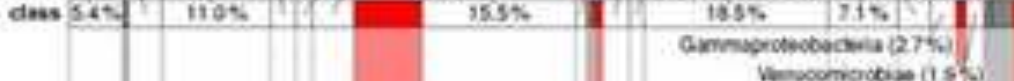

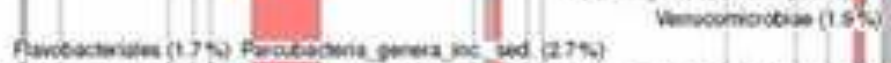

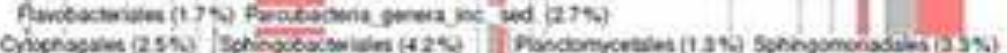

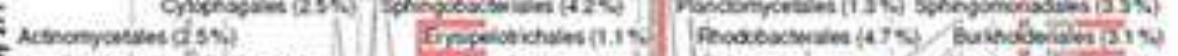

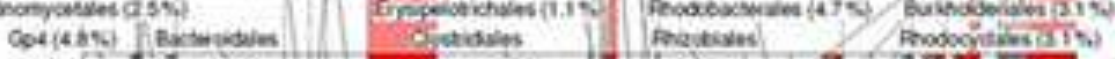

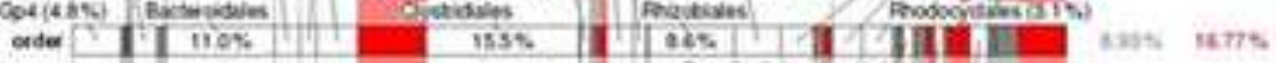

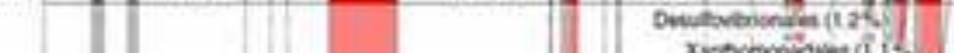

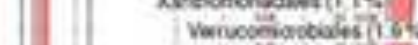

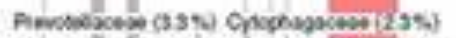

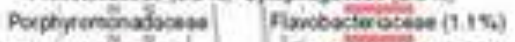

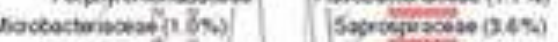

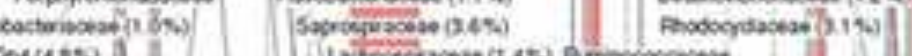

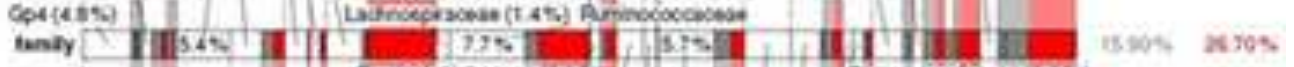
tany IIIsat II ||

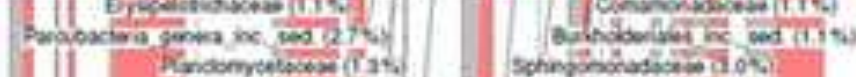

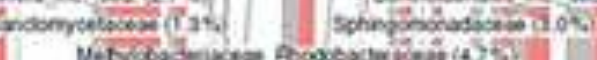

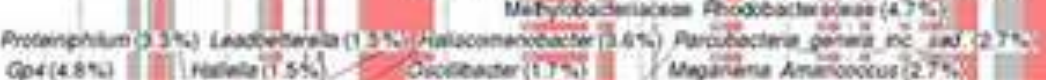

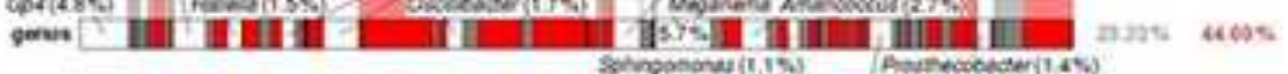

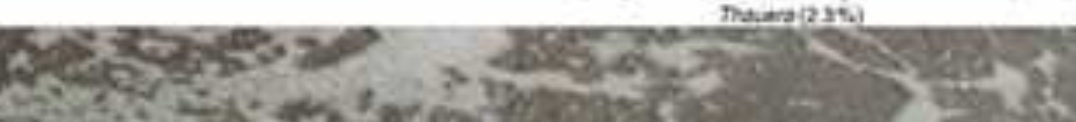
. 